\title{
Self-similarity in the inertial region of wall turbulence
}

\author{
J. Klewicki, ${ }^{1, a)}$ J. Philip, ${ }^{1, \text { b) }}{ }^{1}$. Marusic, ${ }^{1, c)}$ K. Chauhan, ${ }^{1, d)}$ and C. Morrill-Winter ${ }^{1, e)}$ \\ ${ }^{1}$ Department of Mechanical Engineering, The University of Melbourne,
} Victoria 3010 Australia

(Dated: 10 October 2014)

The inverse of the von Kármán constant, $\kappa$, is the leading coefficient in the equation describing the logarithmic mean velocity profile in wall bounded turbulent flows. Klewicki (J. Fluid Mech., vol. 718, 2013 , p. 596) demonstrates that the asymptotic value of $\kappa$ derives from an emerging condition of dynamic self-similarity on an interior inertial domain that contains a geometrically self-similar hierarchy of scaling layers. A number of properties associated with the asymptotic value of $\kappa$ are revealed. This is accomplished using a framework that retains connection to invariance properties admitted by the mean statement of dynamics. The development leads toward, but terminates short of, analytically determining a value for $\kappa$. It is shown that if adjacent layers on the hierarchy (or their adjacent positions) adhere to the same self-similarity that is analytically shown to exist between any given layer and its position, then $\kappa \equiv \Phi^{-2}=0.381966 \ldots$, where $\Phi=(1+\sqrt{5}) / 2$ is the golden ratio. A number of measures, derived specifically from an analysis of the mean momentum equation, are subsequently used to empirically explore the veracity and implications of $\kappa=\Phi^{-2}$. Consistent with the differential transformations underlying an invariant form admitted by the governing mean equation, it is demonstrated that the value of $\kappa$ arises from two geometric features associated with the inertial turbulent motions responsible for momentum transport. One nominally pertains to the shape of the relevant motions as quantified by their area coverage in any given wall-parallel plane, and the other pertains to the changing size of these motions in the wall-normal direction. In accord with self-similar mean dynamics, these two features remain invariant across the inertial domain. Data from direct numerical simulations and higher Reynolds number experiments are presented and discussed relative to the self-similar geometric structure indicated by the analysis, and in particular the special form of self-similarity shown to correspond to $\kappa=\Phi^{-2}$.

\footnotetext{
a) Also at the Department of Mechanical Engineering, University of New Hampshire, Durham, NH 03824 USA.; Electronic mail: klewicki@unimelb.edu.au. or joe.klewicki@unh.edu

b) Electronic mail: jimmyp@unimelb.edu.au.

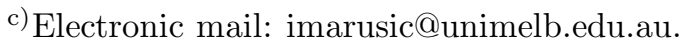

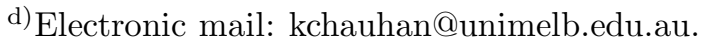

e) Electronic mail: caleb.morrillwinter@gmail.com. 1
} 


\section{INTRODUCTION}

The present analysis considers fully developed and statistically stationary turbulent flows driven by a pressure gradient in circular pipes and planar channels, and the flow in the developing zero pressure gradient boundary layer. As described in standard textbooks on

turbulence, ${ }^{1-3}$ the pipe and channel flow profiles are one-dimensional in the mean. The boundary layer profiles are two-dimensional in the mean, but with their most rapid variation in the direction normal to the wall. In this case, the second dimension derives from the boundary layer thickness exhibiting slow growth in the streamwise direction. The focus herein is on the asymptotic behaviors of the mean velocity profiles in these canonical flows, and by virtue of this, the mean vorticity and Reynolds stress profiles as well. In accord with convention, the main flow is in the positive $x$ direction, with the $y$ coordinate normal to the wall, $y=0$ denoting the wall location. The mean velocity in $x$ is given by $U$, while the fluctuating $x$ and $y$ components are given by $u$ and $v$, respectively. The boundary layer thickness, half channel height and pipe radius are all denoted by $\delta$, and the long time average is denoted by angle brackets.

Over an interior domain between the wall and the position of maximum velocity the mean velocity profile in the flows of interest closely adheres to an equation of the form

$$
U^{+}=\frac{1}{\kappa} \ln \left(y^{+}\right)+B
$$

where the superscript ' + ' denotes normalization by the kinematic viscosity, $\nu$, and the friction velocity $u_{\tau}=\sqrt{\tau_{w} / \rho}$ (e.g., $U^{+}=U / u_{\tau}$ and $y^{+}=y u_{\tau} / \nu$ ), with $\tau_{w}$ being the mean wall shear stress and $\rho$ the mass density of the fluid. A wealth of experimental data reveals that the logarithmic dependence of (1) generically holds, while the value of $B$ changes depending on factors such as surface roughness. The leading coefficient in (1) is the inverse of the von Kármán constant, $\kappa$. Its numerical value, and its physical and mathematical origins are of central interest in the present study.

The literature pertaining to the logarithmic behavior of the mean profile is vast. For an entrée into this body of research one is first referred to standard textbooks, ${ }^{1-3}$ and then to recent reviews on turbulent wall-flow structure and its Reynolds number scaling. ${ }^{4-6}$ As surveyed by Klewicki, ${ }^{5}$ since the early part of the 20th century a variety of semi-analytical, phenomenological, and semi-empirical methods have been used to rationalize the empirical 
observation of a logarithmic profile that is well-approximated by (1). These methods include $(i)$ those that invoke hypotheses associated with a mixing length, ${ }^{7-11}$ ( $\left.i i\right)$ a variety of approaches that assume a two-length scale structure and the existence of an intermediate region (the so-called overlap layer) where the mean profile is presumed to be simultaneously a function of the inner-normalized wall-normal coordinate $\left(y^{+}\right)$and the outer-normalized coordinate $(\eta=y / \delta),{ }^{12-20}$ and $(i i i)$ those that, in accord with dimensional considerations, incorporate the existence of an interior region where the only characteristic length scale is the distance from the wall, $y$, itself. ${ }^{21-25}$

An objective criticism of the approaches just mentioned is that none of them retains firm connection to the relevant mean statement of dynamics, i.e., the Reynolds averaged form of the Navier-Stokes equation. The reason for this is traceable to the nonlinearities inherent to the time-rate-of-change of momentum terms (i.e., inertial terms) in the Navier-Stokes equations, which, when time averaged, render the mean dynamical equation indeterminate. This is the so-called closure problem. ${ }^{1-3}$ The strategy typically employed to overcome the closure problem has been to invoke additional assumptions, or wholly new hypotheses, in order to make the problem tractable. This is the case for all of the approaches noted above. A second and related criticism is that, while such approaches enjoy a degree of success in describing what behaviors are observed, they are challenged relative to describing how and why these behaviors occur. ${ }^{35}$ These challenges also follow from their lack of connection to the governing equations. In particular, all of the above approaches incorporate the von Kármán constant, but none are equipped to elucidate its physical or mathematical origins. Accordingly, research investigations associated with $\kappa$ have primarily focused on empirically estimating its numerical value. ${ }^{26-30}$

In contrast to earlier approaches, the analytical framework employed herein retains a grounding in the mean dynamical equation. For this reason its findings speak directly to the solution properties admitted by this equation. The present approach determines an invariant form admitted by the mean momentum equation. This underlies the development of an analytical closure that becomes increasingly accurate as the Reynolds number becomes large, e.g., see Appendix A for a derivation of the logarithmic mean velocity profile directly from the mean momentum equation. This closure leverages the self-similarities formally admitted by the mean momentum equation, revealing the emergence of a genuine similarity solution ${ }^{37}$ on the inertial subdomain where (1) is empirically seen to hold. ${ }^{29}$ The coordinate stretching 
function that underlies the invariance of this similarity solution is of central importance to the present analyses, as its functional dependencies describe the geometric properties of the flow associated with the asymptotic value of $\kappa$. More broadly relevant to methods of mathematical physics, the present approach has apparent connection to other studies that have exploited the finite and differential transformations (scaling functions) underlying a similarity solution for the purpose of exposing solution properties in an asymptotic regime, including those that terminate with a singularity, e.g., Zeff et al. ${ }^{31}$

Herein the focus is on $\kappa$ and the self-similar structure that underlies its asymptotic value. In this regard, it is useful to note that the conditions of large inner-normalized distance from the wall $\left(y^{+}=y u_{\tau} / \nu \rightarrow \infty\right)$ and correspondingly, large Reynolds number $\left(\delta^{+}=\delta u_{\tau} / \nu \rightarrow\right.$ $\infty)$, are implicit to the notion of $\kappa$ having a universal constant value. In what follows we first describe the salient properties admitted by the mean momentum equation. This provides the groundwork for the subsequent analysis that, among other things, naturally leads us to suspect that $\kappa=\Phi^{-2}=0.381966 \ldots$, where $\Phi=(1+\sqrt{5}) / 2$ is the golden ratio. We then explore what these analyses tell us about the self-similar structure in the inertial region of turbulent wall flows as $y^{+} \rightarrow \infty$.

\section{ANALYTICAL FRAMEWORK}

The analytical framework derives from the body of work initiated by Fife and coworkers, ${ }^{32-35}$ and continued by Klewicki and co-workers. ${ }^{36-39}$ The mean velocity profile is a solution to the mean momentum equation as constrained by the relevant boundary conditions. Thus, the properties of interest reflect the structure of the mean dynamics. Many of the results needed to serve the present objectives are in the existing archival literature. The aim of this section is to collect and describe these results.

We use the channel configuration since it is the most straight-forward to describe. The inner-normalized mean momentum equation for turbulent channel flow is

$$
\begin{aligned}
& 0=\frac{1}{\delta^{+}}+\frac{d^{2} U^{+}}{d y^{+2}}+\frac{d T^{+}}{d y^{+}} \\
& 0=\mathrm{PG}+\mathrm{VF}+\mathrm{TI}
\end{aligned}
$$

where $T^{+}=-\langle u v\rangle / u_{\tau}^{2}=-\langle u v\rangle^{+}$is often referred to as the Reynolds stress. Here it is also 
relevant to note that when the origin is shifted from the pipe centerline to the wall the mean momentum equation for pipe flow is identical to (2). The terms in (2) represent the mean pressure gradient $(\mathrm{PG})$, the mean viscous force $(\mathrm{VF})$ and the net mean effect of turbulent inertia (TI). It is this last term that arises from time averaging the nonlinear inertial terms, as discussed previously. All the terms in (2) are of leading order somewhere in $0 \leq y \leq \delta$, but are not necessarily leading order everywhere.

The multiscale analysis employs the framework established by Fife et al., ${ }^{33,35}$ which provides a means of identifying solution properties and scaling behaviors (if they exist) of indeterminate equations. The framework employs generic mathematical criteria, and thus is not wedded to any particular physical problem. These criteria consist of admissibility and compatibility conditions for the existence of what is called a scaling patch. A scaling patch is an interval of the solution domain where there exists a differential scaling such that the governing differential equation can be written in a parameter free invariant form that reflects the actual leading order balances of terms for variations of the governing parameter(s). For example, if applied to the two-dimensional, flat plate, laminar boundary layer flow, the present method correctly identifies the entire width of the flow as a single scaling patch, and that the relevant differential transformations are those first identified by Blasius. ${ }^{40}$ An admissible scaling is a differential scaling for which the governing equation has at least two terms of nominal order of magnitude 1, where nominal refers to the order indicated by the given normalization. The admissibility condition ensures the existence of meaningful leading order dynamics.

The main assumption of the method is as follows. Given an admissible scaling and a point $y_{0}$ in the solution domain, consider the set of all derivatives appearing in the normalized form of the governing equation that have nominal order 1 , evaluated at a given point, $\hat{y}=0$. Here $\hat{y}$ is the normalized variable associated with the given scaling patch. If each derivative in the set is known to be numerically $\leq O(1)$ and there exists a derivative, not necessarily in that set, which is $O(1)$, then that scaling, together with some interval containing $y_{0}$, is a scaling patch. This is the compatibility criterion. The condition that one derivative be $O(1)$ is to prevent trivial scalings where, for example, the normalized dependent variables do not change under variations in the normalized independent variable. Note that the main assumption constitutes a minimal requirement, since normalizations that do not satisfy it will not yield an invariant profile on the given scaling patch when the parameter(s), e.g., 
Reynolds number, are varied. Empirical data are used in the method to verify that the leading order terms, as nominally determined from rescaling the governing equation on a given scaling patch, are indeed the actual leading order terms. This is generally an attainable data requirement, since one only needs to determine the relative magnitudes of the terms. Once the leading terms are determined, the rest of the analysis relies on the governing equation and its boundary conditions.

No claims are made regarding the uniqueness of the mean solutions found by the method. To date, however, all of the resulting predictions exhibit remarkable agreement with the mean solutions realized via direct numerical simulations (DNS) or experiments, e.g. ${ }^{32,36-38}$ For the present problem of turbulent channel flow $T^{+}\left(=-\langle u v\rangle^{+}\right)$is assumed to take on positive values. This assumption receives universal empirical support. Lastly, when employing the transformations that lead to an invariant form of (2), aspects of the continuing analysis require that a decision be made regarding the parameter $\lambda$ that appears in (7). As in Fife et al., ${ }^{35} \lambda$ is herein taken to equal 1 . This is because $(i) \lambda=1$ corresponds to the simplest set of transformations that yield an invariant mean equation, (ii) existing empirical data uniformly indicate that the velocity increment across layer III is $O\left(u_{\tau}\right)$ independent of $\delta^{+}$, and this can only occur if $\lambda=1$, and concomitantly, (iii) $\lambda=1$ is the only condition that asymptotically yields a logarithmic $U^{+}\left(y^{+}\right)$solution to (2). Succinctly, if $\lambda \neq 1$ the entire notion of $\kappa$ loses relevance.

The analysis proceeds from a knowledge of the leading order terms, including the scaling behaviors of the domains over which these leading order balances exist. This information has been analytically determined and empirically verified using the method just described. The resulting leading order balances and associated layer scaling properties are summarized in table I. The layer structure associated with the leading order balances in (2) is also depicted in figure 1.

The mean momentum equations for the canonical turbulent wall-flows asymptotically admit an invariant form over an interior domain that resides between the inner and outer peak positions ( $y_{p i}$ and $y_{p o}$, respectively) of the TI term in (2), see figure 2. On this domain, TI is a strictly decreasing function that exhibits a diminishing but non-negligible rate of change with increasing distance from the wall. ${ }^{33}$ The invariant form of (2) investigated thus far (there may be others) derives from stretching the derivative of the TI term as a function of $y$. Specifically, the quantity 


\begin{tabular}{ccccc}
\hline \hline Physical & Magnitude ordering & Magnitude ordering & $\Delta y$ increment & $\Delta U$ increment \\
layer & (pipe \& channel) & (boundary layer) & & \\
I & $|\mathrm{PG}| \simeq|\mathrm{VF}| \gg|\mathrm{TI}|$ & $|\mathrm{MI}| \simeq|\mathrm{VF}| \gg|\mathrm{TI}|$ & $O\left(\nu / u_{\tau}\right)(\leq 3)$ & $O\left(u_{\tau}\right)(\leq 3)$ \\
II & $|\mathrm{VF}| \simeq|\mathrm{TI}| \gg|\mathrm{PG}|$ & $|\mathrm{VF}| \simeq|\mathrm{TI}| \gg|\mathrm{MI}|$ & $O\left(\sqrt{\nu \delta / u_{\tau}}\right) \quad(\simeq 1.6)$ & $O\left(U_{e}\right)(\simeq 0.5)$ \\
III & $|\mathrm{PG}| \simeq|\mathrm{VF}| \simeq|\mathrm{TI}|$ & $|\mathrm{MI}| \simeq|\mathrm{VF}| \simeq|\mathrm{TI}|$ & $O\left(\sqrt{\nu \delta / u_{\tau}}\right)(\simeq 1.0)$ & $O\left(u_{\tau}\right)(\simeq 1)$ \\
IV & $|\mathrm{PG}| \simeq|\mathrm{TI}| \gg|\mathrm{VF}|$ & $|\mathrm{MI}| \simeq|\mathrm{TI}| \gg|\mathrm{VF}|$ & $O(\delta)(\rightarrow 1)$ & $O\left(U_{e}\right)(\rightarrow 0.5)$ \\
\hline \hline
\end{tabular}

TABLE I. Magnitude ordering and scaling behaviors associated with the four layer structure of the mean dynamics. ${ }^{32,35}$ Note that $U_{e}$ equals $U_{\infty}$ in the boundary layer and $U_{c}$ in the pipe and channel. MI refers to the mean inertia term that appears in the mean boundary layer equation.

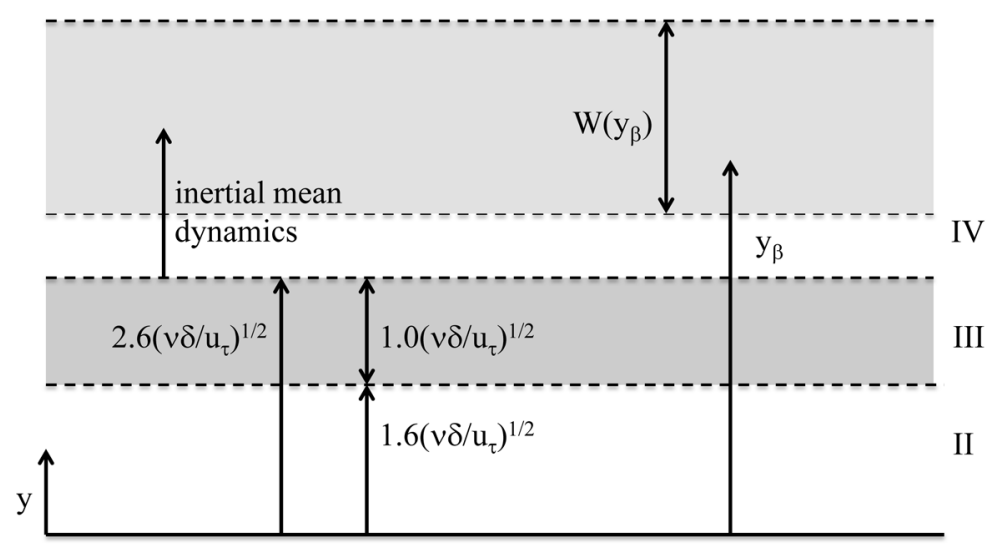

FIG. 1. Schematic of the layer structure associated with the leading order balance of terms in (2), and a representative layer on the underlying hierarchy of layers. The value of $\beta$ uniquely corresponds to a wall-normal distance, $y_{\beta}^{+}$. Every $y_{\beta}^{+}$is straddled by a layer having a width, $W^{+}\left(y_{\beta}\right)$, that becomes proportional to $y_{\beta}^{+}$as $y^{+} \rightarrow \infty$.

$$
A=-\frac{d^{2} \hat{T}}{d \hat{y}^{2}}=-\frac{d^{2} T^{+}}{d y^{2+}}\left(\frac{d T^{+}}{d y^{+}}+\frac{1}{\delta^{+}}\right)^{-3 / 2}=-\frac{d^{2} T^{+}}{d y^{2+}} \beta^{-3 / 2}
$$

becomes an $O(1)$ function on a continuous hierarchy of self-similar scaling layers. Moreover, as $\delta^{+} \rightarrow \infty, A$ approaches constancy on the inertial subdomain of the layer hierarchy where $\mathrm{TI} \simeq \mathrm{PG}$. Under this condition, the stretching given by (3) maps the decreasing curvature $T^{+}$profile onto an invariant, constant curvature, $\hat{T}$ profile. The subdomains where $A \rightarrow$ const. and where $A$ is $O(1)$ but non-constant are indicated in figure 2 . 


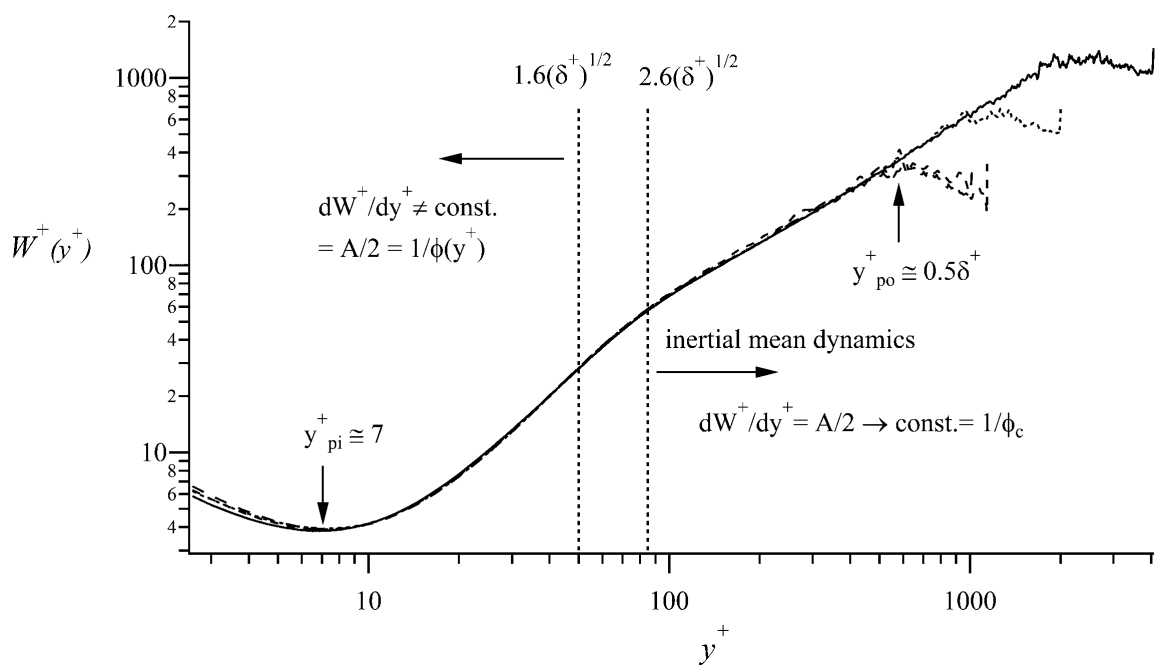

FIG. 2. Distribution of $W^{+}\left(y^{+}\right)$for pipe and channel flows; pipe flow DNS ${ }^{41}$ at $\delta^{+}=1142$, - - channel flow $\mathrm{DNS}^{42}$ at $\delta^{+}=1020,---$; channel flow $\mathrm{DNS}^{43}$ at $\delta^{+}=2004, \cdots \cdots$; channel flow $\mathrm{DNS}^{11}$ at $\delta^{+}=4080, \longrightarrow$. Vertical lines denoting the beginning and end of layer III are computed for $\delta^{+}=1000$ (see figure 1). The upper and lower bounds of the layer hierarchy correspond to the inner $\left(y_{p i}\right)$ and outer $\left(y_{p o}\right)$ peaks of the TI term, or equivalently the minimum and maximum values of $W^{+}\left(y^{+}\right)$.

As shown by the last equality in (3), the 'hat' indicates normalization using $u_{\tau}$ and the continuously varying length scale function,

$$
W^{+}\left(y^{+}\right)=O\left(\beta^{-1 / 2}\right),
$$

that quantifies the inner-normalized widths of the layers on the hierarchy. Note that one can take $W^{+}=\beta^{-1 / 2}$ without penalty, since $\beta^{-1 / 2}$ is what is actually used in calculations. Note also that $A$ derives from a ratio involving the wall-normal derivatives of $T^{+}$, and that the self-similar condition of present interest occurs when $A \rightarrow$ const. The fact that this condition solely relates to the rates of change of $T^{+}\left(y^{+}\right)$is central to understanding how and why self-similarity is attained (approximated) on a domain that is sufficiently remote from the imposed length scales associated with the boundary conditions. The second equality in (3) indicates that $W^{+}\left(y^{+}\right)$varies inversely with the square root of the TI term in the mean momentum balance. ${ }^{33}$ As a practical matter, and by virtue of $(2), W^{+}\left(y^{+}\right)$is typically most accurately computed using $W^{+}=\left(-d^{2} U^{+} / d y^{+2}\right)^{-1 / 2}$, e.g., see Ref. 36. Owing to its 
direct relationship to the TI term, $W^{+}\left(y^{+}\right)$is physically recognized as the average size of the motions responsible for the net wallward flux of momentum from layer to layer, or, equivalently, the average size of the motions responsible for the generation of $T^{+}=-\langle u v\rangle^{+}$. (Figure 6 provides direct evidence that strongly supports this assertion.) Distributions of $W^{+}\left(y^{+}\right)$at Reynolds numbers accessible to direct numerical simulations (DNS) are shown in figure 2 . $W^{+}$becomes directly proportional to $y^{+}$on the hierarchy as $y^{+} \rightarrow \infty$ (Ref. 35, p. 796).

Dynamically, $A \rightarrow$ const. indicates a constant flux of turbulent inertial 'force' from layer to layer on the hierarchy. How this occurs is reflected in the invariant form of (2)

$$
\frac{d^{2} U^{+}}{d \hat{y}^{2}}+\frac{d \hat{T}}{d \hat{y}}+1=0 .
$$

This equation is attained by using the differential transformations,

$$
d y^{+}=W^{+} d \hat{y}, \quad d \hat{T}=W^{+} d T^{+}, \quad d U^{+}=d \hat{U}
$$

that have associated finite transformations,

$$
y^{+}=y_{\beta}^{+}+\beta^{-1 / 2} \hat{y}, \quad T^{+}=T_{m}^{+}\left(y_{\beta}^{+}\right)+\beta^{1 / 2} \hat{T}(\hat{y}), \quad U^{+}=U^{+}\left(y_{\beta}^{+}\right)+m\left(y_{\beta}^{+}\right)\left(y^{+}-y_{\beta}^{+}\right)+\lambda \hat{U}(\hat{y}) .
$$

Between (6) and (7) $W^{+}$and $\beta^{-1 / 2}$ are used interchangeably to emphasize their equivalence. In (7) $m$ is the mean velocity gradient, $d U^{+} / d y^{+}$, at $y^{+}=y_{\beta}^{+}$, where $y_{\beta}^{+}$identifies the location of each $W^{+}$layer. That is, while the differential transformations are sufficient to obtain (5) (Ref. 35, p. 796), the finite transformations reveal the layer structure depicted in figure 3 .

For future reference we note that (7) indicates that the 'hat' variables are defined locally on each member of the hierarchy of layers, but since $W^{+}\left(y^{+}\right)$varies continuously (5) holds everywhere on the hierarchy (see Appendix A). This equation shows that across each layer of width $W^{+}$all three terms are $O(1)$, and thus the exchange of the leading order balance of terms in (2) that occurs across layer III (see table I) is self-similarly replicated across each of the hierarchy layers. This self-similarly replicating geometric structure is depicted for a few $W^{+}$in figure 3. Lastly, note that across any $W^{+}$the velocity increment, $\Delta U^{+}$, is $O(1)$, and becomes constant as $y^{+} \rightarrow \infty$, i.e., as $\phi \rightarrow \phi_{c}$ (Ref. 35, p. 797). This fact is employed below. 


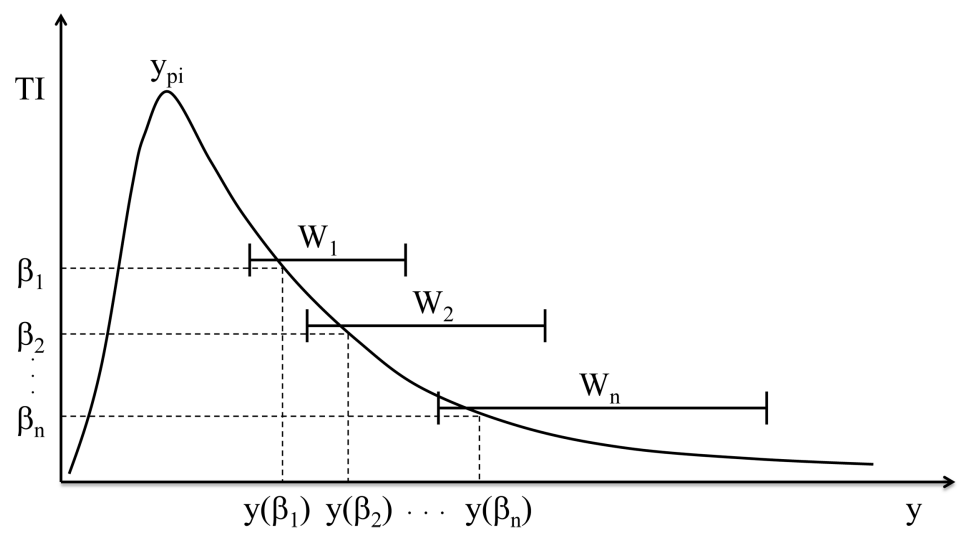

FIG. 3. Schematic depiction of the layer hierarchy described by the continuous distribution of widths, $W\left(y^{+}\right)$. Note that when each member of $W$ and $u_{\tau}$ are used to normalize (2), the result is (5) which holds exactly.

Similarity solutions arise when the relevant governing equation admits an invariant form. ${ }^{44}$ Over the domain of the layer hierarchy (2) exhibits two types of self-similarity. ${ }^{37}$ Dynamically, these are associated with the behaviors of $A$ just described. In addition, it has been shown (Ref. 36, p. 89) that $A / 2=d W / d y$. This finding connects the geometry of the hierarchy to the underlying mean dynamics, and reveals a basis for distance from the wall scaling that follows from the invariance properties of (2). To see this, recall that $A \rightarrow$ const. physically describes the flux of turbulent force from one layer to the next across the hierarchy, while $A / 2=d W / d y$ indicates that $W$ approaches direct proportionality with $y$ as $\delta^{+} \rightarrow \infty$. As noted by Fife et al. ${ }^{35}$ (p. 798), the constancy of $A$ can only be disrupted by effects from adjacent layers on the continuous hierarchy, and thus ultimately only from effects acting at the periphery of the hierarchy. Thus, as seen in other contexts, ${ }^{45}$ self-similarity emerges on an interior domain that is sufficiently remote from the overall flow boundaries as $\delta^{+} \rightarrow \infty$.

Similarity solutions are associated with a coordinate stretching function that preserves the invariance of the solution for variations in the relevant parameter(s), in this case $\delta^{+}$. For (2) this stretching function is given by

$$
\frac{d W}{d y}=\phi^{-1}=\frac{A}{2} .
$$

Physically, $\phi$ is the stretching of the $y$ coordinate required to produce an invariant represen- 
tation of the flux of turbulent force as generated (on average) by the momentum transport of $W$ sized eddies (see equation 3). Note that like $A$ and $d W / d y, \phi$ is $O(1)$ but varies for $y^{+} \lesssim 2.6 \sqrt{\delta^{+}}$, and approaches a constant, $\phi=\phi_{c}$, on the inertial domain of interest. (Here we note that Klewicki ${ }^{37}$ chose the Greek letter $\phi$ for the coordinate stretching function to acknowledge Prof. Paul Fife for his developments of the theory described herein. It is thus purely a coincidence that on the relevant domain the symbol used for the Fife similarity parameter, $\phi$, is the same commonly used for the golden ratio, $\Phi$.) By virtue of (8), (2) has been shown to admit a similarity solution on the inertial domain where the mean profile most rapidly develops logarithmic dependence. On the domain $\left(2.6 \sqrt{\delta^{+}} \lesssim y^{+} \lesssim 0.3 \delta^{+}\right)$, this solution for both $U^{+}$and $T^{+}$varies from the $\delta^{+}=2004$ channel DNS ${ }^{43}$ by less than $0.1 \%$; even though at this low Reynolds number $U^{+}\left(y^{+}\right)$noticeably deviates from purely logarithmic dependence (Ref. 37, p. 613). These findings provide a rational basis for recent high Reynolds number observations indicating that the mean velocity asymptotically exhibits logarithmic dependence on this domain, ${ }^{29}$ and potentially for the logarithmic dependence of the higher order even moments of $u$ on this domain as well. ${ }^{46}$ Here we note, for example, that approaches that invoke the distance from the wall scaling assumption (discussed in the Introduction) provide no information pertaining to the bounds of the domain where logarithmic dependence is expected, while $y^{+}=O\left(\sqrt{\delta^{+}}\right)$is in the center of the classically defined overlap layer, rather than at its lower boundary as indicated by the data.

The analysis of Fife et al. ${ }^{35}$ shows that at any finite $\delta^{+}(2)$ has the approximate solution,

$$
U^{+}=\phi^{2} \ln \left(y^{+}-C\right)+B
$$

on the inertial domain, and that this solution becomes exact as $\delta^{+} \rightarrow \infty$, i.e., as $\phi \rightarrow \phi_{c}$. For later reference, we also note that the analysis leading to (9) reveals that there are two physical effects associated with the leading coefficient in (9). We will return to this in $§ I V$. For completeness, the analytical steps leading to (9) are presented in Appendix A.

As predicted by the analysis, figures 2 and 6 provide convincing evidence that with increasing Reynolds number $W^{+}\left(y^{+}\right)$is increasingly well-approximated by the linear function,

$$
W^{+}\left(y^{+}\right)=\frac{y^{+}-C}{\phi_{c}},
$$

on the portion of the hierarchy where the leading order mean dynamics are wholly inertial. 


\section{PROPERTIES RELATING TO $\kappa$}

Invariant mean dynamics and the self-similar geometric structure they generate are used to reveal a number of properties associated with the value of $\kappa$ as $\delta^{+} \rightarrow \infty$, and to clarify the mean dynamical structure on the inertial domain of interest.

\section{A. Geometric Structure}

The geometry of the layer structure depicted in figure 1 stems from the condition (3). This condition is attained on each layer of width, $W^{+}$, that contains the position, $y_{\beta}^{+}$, where the scaled value of the transformed Reynolds stress, $\hat{T}$, is maximal (Ref. 34, p. 175). Thus, as depicted in figure $3, y_{\beta}^{+}$resides within the bounds of its associated $W^{+}$. When considering a specific representation of the hierarchy there are, however, a number natural choices regarding where to anchor the origin of each layer. ${ }^{34}$ These include where the balance exchange of the terms in (5) begins, and where the TI contribution on each layer crosses zero. These are equally valid, since, by definition, these points remain within the bounds of $\pm O\left(W^{+}\right)$for all $\delta^{+}$. Note further that the actual layer hierarchy is continuous, and thus adjacent layers have considerable overlap. Because the local $W$ and $u_{\tau}$ normalization is valid over a finite domain, one may also choose to employ a discrete representation of the layer hierarchy, ${ }^{34}$ with the number of layers becoming countably infinite as $\delta^{+} \rightarrow \infty$.

Given these considerations, the discrete layer hierarchy construction depicted in figure 4 is employed, as this facilitates a convenient way to analytically (algebraically) and graphically express the relationship between adjacent layers and their positions. This self-consistent representation places the reference point at the beginning of each discrete layer. Note that the relationship from one layer to the next is that $y_{2}^{+}$is $W_{1}^{+}$greater than $y_{1}^{+}$and so forth. This representation retains validity since the layer structure preserves the proportional relationship between the $y_{i}^{+}$and $W_{i}^{+}(10)$, and on each $W^{+}$the normalized values of mean velocity and Reynolds stress $\left(U^{+}\right.$and $\hat{T}$, respectively) and their derivatives with respect to $\hat{y}$ remain $\leq O(1)$ but greater than zero as $y^{+} \rightarrow \infty$. The validity of the representation in figure 4 is corroborated in section IIIB via use of the continuous transformations underlying (5), and the presentation in Appendix B provides additional evidence that, when defined as the zero-crossing in the TI term, the $y_{\beta}^{+}$move to the beginning of each layer as $\delta^{+} \rightarrow \infty$. 
Self-similarity in the inertial region of wall turbulence

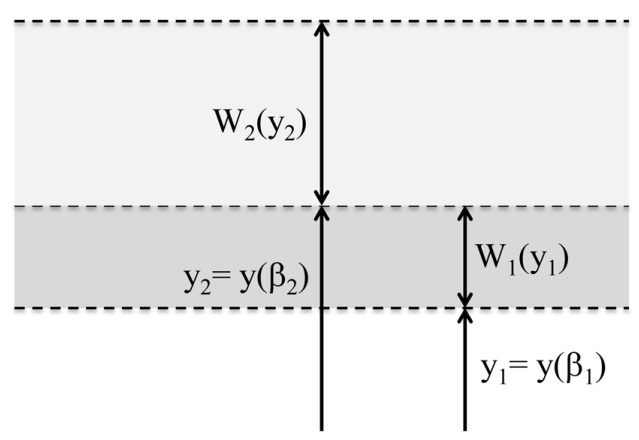

FIG. 4. Schematic of the layer structure used in the present geometric analysis. Here the anchor for each layer of width $W$ is located at the beginning of that layer.

The geometric implications of the asymptotic layer structure are now described. Beginning with (10) and neglecting the constant as $y^{+} \rightarrow \infty$ leads to $y_{1}=\phi_{c} W_{1}, y_{2}=\phi_{c} W_{2}$, and thus

$$
\frac{W_{1}}{W_{2}}=\frac{y_{1}}{y_{2}}
$$

Invoking $y_{1}+W_{1}=y_{2}=\phi_{c} W_{1}+W_{1}$, cross multiplying and solving for $W_{2} / W_{1}$ yields

$$
\frac{W_{2}}{W_{1}}=\frac{\phi_{c}+1}{\phi_{c}}=\frac{y_{2}}{y_{1}} .
$$

From the above geometric considerations the $W_{i}$ and the $y_{i}(i=1 \ldots n)$ are recognized as members of two interlaced geometric sequences. For both sequences the ratio of adjacent terms (the common ratio) is equal to $\left(\phi_{c}+1\right) / \phi_{c}$. These findings are succinctly summarized by

$$
\phi_{c}=\frac{y_{1}}{W_{1}}=\frac{y_{2}}{W_{2}}=\alpha \frac{y_{2}}{y_{1}}
$$

with proportionality constant $\alpha=\phi_{c}^{2} /\left(\phi_{c}+1\right)$ that derives from

$$
\frac{y_{2}}{y_{1}}=\frac{W_{1}}{y_{1}} \frac{W_{2}}{W_{1}} \frac{y_{2}}{W_{2}}=\left(\frac{\phi_{c}+1}{\phi_{c}^{2}}\right) \phi_{c}
$$

Note that if $\alpha=1$, then the (physically relevant) positive root for $\phi_{c}$ is $(1+\sqrt{5}) / 2=\Phi$. 


\section{B. Dynamical Structure}

The changes in momentum from layer to layer are clarified by using the previously noted result that the inner-normalized velocity increment across each $W^{+}$in the inertial domain is $O(1)$ and becomes a constant as $y^{+} \rightarrow \infty$. Beginning with the asymptotic form of (9) and neglecting the offset $C$ for large $y^{+}$yields

$$
\ln \left(y_{2}^{+}\right)-\ln \left(y_{1}^{+}\right)=D \phi_{c}^{-2}
$$

where $D$ is the constant velocity increment, and consistent with $(8), \phi_{c}^{2}$ is the leading coefficient in (9). Rearranging the logarithms and exponentiating gives

$$
\frac{y_{2}}{y_{1}}=e^{D \phi_{c}^{-2}}=G=\frac{\phi_{c}+1}{\phi_{c}}=\text { const. }
$$

Employing (11) and (12) yields

$$
G y_{1}-y_{1}=W_{1}=y_{1} / \phi_{c}
$$

or

$$
G-1=\phi_{c}^{-1}=(\alpha G)^{-1}
$$

Note that if $\alpha=1, G=\phi_{c}=\Phi$.

We now verify that the value of $D$ determined from the construction of figure 4 is indeed invariant. This is done by recasting (16) into its similarity variable form, and examining its behavior as $y^{+} \rightarrow \infty$. By once integrating (2), using the equation for $T^{+}$derived by Fife et al. ${ }^{34}$ (also see Appendix A) and employing the boundary conditions, it is a straightforward matter to show that

$$
\frac{d U^{+}}{d y^{+}}=\frac{\phi_{c}^{2}}{y^{+}}
$$

on the self-similar inertial domain of interest. Noting that $\kappa=\phi_{c}^{-2}$, (19) is also recognized as the familiar representation of the mean velocity gradient that leads to the logarithmic mean profile given by (1).

Here we note that the present formulation connects to that of Barenblatt et al. ${ }^{15}$ in the following manner. Through the use of dimensional analysis they arrive at (19), but then note 
that from dimensional considerations alone there is no compelling reason to assume that the parameter, $\kappa^{-1}=\phi_{c}^{2}$ (which is in general a function of $y^{+}$and $\delta^{+}$), should attain a nonzero constant value as $y^{+}$and $\delta^{+}$tend to infinity. The present theory also indicates that $\phi$ is expected to vary at low $\delta^{+}$. As opposed, however, to dimensional considerations alone, the present theory reflects the properties associated with the invariant form (5), and in particular the condition (3). Owing to this, more can be discerned about $\phi$. Namely, $\phi$ is known to be a non-zero $O(1)$ function, and based upon the analytical construction, is expected to approach constancy as $y^{+} \rightarrow \infty .{ }^{35}$ Given (8), the data of figure 2 provide compelling empirical support for this expectation. In this regard, existing evidence also suggests that for $\delta^{+}$values as low as about 1000 the intrinsic self-similarity that underlies the existence of logarithmic dependence is well-approximated, even though $\phi$ is quite different from $\phi_{c}$. This connects to the observation that at low Reynolds number $d W / d y$ is approximately linear ( $\phi \simeq$ const), but the slope of this approximately linear variation changes with increasing Reynolds number. ${ }^{37}$

By employing the differential transformation for $d \hat{y}(6)$ on the left of (19), and the finite transformation for $\hat{y}(7)$ on the right, one obtains

$$
\frac{d U^{+}}{d \hat{y}}=\frac{\phi_{c}^{2}}{\hat{y}_{\beta}+\hat{y}}
$$

where $\hat{y}_{\beta}=\beta^{1 / 2} y_{\beta}^{+}$is the anchor position of each $W^{+}$layer, and $\hat{y}$ is the local variable on each $W$, see figures 3 and 4 .

The present purpose is served by integrating (20) with respect to the similarity variable, $\hat{y}=y / W$. Here we leverage the fact that the integral of an invariant function is also invariant, since, by definition, a similarity transformation maps all dimensional solutions onto the single domain described by the similarity variables. Here the primary focus is on the subdomain covering a single $\hat{W}$, since the transition across every dimensional $W$ is mapped onto this. Note also that $\hat{W}=W / W=1$.

The definite integral of interest is thus given by

$$
D=\int_{0}^{1} \frac{d U^{+}}{d \hat{y}} d \hat{y}=\phi_{c}^{2} \int_{0}^{1} \frac{d \hat{y}}{\hat{y}_{\beta}+\hat{y}},
$$

where $D$ is the mean velocity increment across any given $W$, and in particular across the single universal $\hat{W}$. Evaluation of (21) gives 
Self-similarity in the inertial region of wall turbulence

$$
D=\left.U^{+}\right|_{0} ^{1}=\left.\phi_{c}^{2} \ln \left(\hat{y}_{\beta}+\hat{y}\right)\right|_{0} ^{1}
$$

or

$$
D=\phi_{c}^{2} \ln \left(\frac{\hat{y}_{\beta}+1}{\hat{y}_{\beta}}\right) .
$$

But, as $y^{+} \rightarrow \infty\left(\delta^{+} \rightarrow \infty\right) \hat{y}_{\beta}=\beta^{1 / 2} y_{\beta}^{+} \rightarrow \phi_{c}$, and thus one obtains,

$$
D=\phi_{c}^{2} \ln \left(\frac{\phi_{c}+1}{\phi_{c}}\right)
$$

which is invariant on the $\phi \rightarrow \phi_{c}$ portion of the hierarchy. Comparison indicates that (24) identically recovers (15). This demonstrates that the discrete geometric construction of figure 4 is in accord with the continuous transformations used to generate the invariant form of the momentum equation (5).

\section{Summary and Observations}

At present, there is no apparent way to advance the above analysis any further. Given this, it is useful to summarize the origin of this development and what it suggests.

All analytical approaches to describing the profiles of $U^{+}$and $T^{+}$ultimately require additional assumptions, hypotheses or empirical input. This stems directly from time averaging the Navier Stokes equation; a process that obscures the physics of the unclosed TI term in (2). The present framework uses empirical information at its outset to verify the leading order balances given in table I. With these leading order balances determined, the remainder of the analysis follows from the boundary value problem described by the mean momentum equation and its boundary conditions. This includes the analytical determination (to within order of magnitude) of the scaling properties reflected in table I. Note further that because the analysis is founded in the properties of the mean flow boundary value problem, in the absence of a change in boundary conditions or additional forces, these scaling behaviors are retained to arbitrarily high Reynolds number, e.g., see Appendix A of Ref. 37. This description characterizes the degree to which the present findings are grounded in the solution properties of (2). 
The data presentation of the following section considers implications of the above analysis relative to the analytically known property that $\phi \rightarrow \phi_{c}=1 / \sqrt{\kappa}$. In this regard, if the widths of adjacent layers (or the positions of adjacent layers) on the $\phi \rightarrow \phi_{c}$ part of the hierarchy follow the same proportionality as each layer width to its position, then

$$
\alpha=\frac{\phi_{c}^{2}}{\left(\phi_{c}+1\right)}=1
$$

holds as $\delta^{+} \rightarrow \infty$. Note that the present theory indicates that the properties of flow statistics on the inertial layer derive from the asymptotically self-similar structure admitted by (2), independent of the value of $\alpha$. Consistently, $\alpha \rightarrow 1$ would mark the emergence of the form of self-similarity just described as $\delta^{+} \rightarrow \infty$. This possibility seems at least plausible given the increasingly self-similar nature of the flow structure on the $\phi \rightarrow \phi_{c}$ domain. Here we reiterate that (25) has only one positive solution, $\phi_{c}=\Phi=(1+\sqrt{5}) / 2$, and thus from (9) yields $\kappa=\Phi^{-2}$. The condition $\alpha=1$ also implies that $\kappa$ obeys

$$
\kappa+\sqrt{\kappa}=1 .
$$

The implication of (25) is that the established analytical result, $y_{i} / W_{i}=\phi_{c}$ (at large $\left.\delta^{+}\right)$, is augmented with $y_{i+1} / y_{i}=W_{i+1} / W_{i}=\phi_{c}$. Relative to traditional wall-turbulence thinking, this constitutes a more expansive form of distance from the wall scaling, and the above analysis shows that if it holds, then $\kappa \equiv \Phi^{-2}$. The dynamical implication of $\alpha=1$ (and thus $\left.\phi_{c}=\Phi\right)$ is that (24) becomes

$$
D=\Phi^{2} \ln (\Phi)=\Phi \ln \left(\Phi^{\Phi}\right) \simeq 1.26
$$

It is emphasized that (25) is, at present, not an analytical finding. Rather, it is the more complete form of asymptotic self-similarity that the above analysis naturally leads us to suspect. Demonstrating that (25) holds (or that $\alpha$ equals any other specific value) would, however, constitute an analytical determination of $\kappa$ to arbitrary precision. Such a determination would be grounded in the mean governing equation to the extent noted above. Appendix C outlines some equivalent mathematical problems associated with analytically determining (25). The following section explores the degree to which empirical evidence supports $\alpha \rightarrow 1$ as $\delta^{+} \rightarrow \infty$, and more broadly, what the present analysis reveals about the self-similar structure of the inertial region of interest. 


\section{EMPIRICAL EVIDENCE AND UNDERLYING SELF-SIMILAR STRUCTURE}

Empirical evidence associated with the scaling properties of the leading order balances of (2) and the location and slope of the logarithmic mean velocity profile are perhaps the two most obvious behaviors to examine. This is then followed by an examination of specific properties that derive from, or are suggested by, elements of the above analysis.

\section{A. Mean Momentum Balance}

Regarding the four layer structure, we note that layer III is a member of the layer hierarchy. ${ }^{34}$ It is, in fact, the central layer of the hierarchy in the geometric mean sense, and this accounts for the $\sqrt{\delta^{+}}$factor in the widths of layers II and III, ${ }^{47}$ also see Appendix D. With this, and the results of table I and figure 1, (25) specifies that $\Delta y_{I I}^{+}=\Phi \sqrt{\delta^{+}}$for the inner-normalized width of layer II, and concomitantly, the analytically determined geometric structure of the layer hierarchy specifies that $\Delta y_{I I}^{+}+\Delta y_{I I I}^{+}=\Phi^{2} \sqrt{\delta^{+}}=(1+\Phi) \sqrt{\delta^{+}}$. Note that this construction directly relates the scaling properties of the mean force balance to the slope constant in the mean velocity profile equation.

Comparison indicates that the empirically estimated layer width values in table I agree with these to within about $1.2 \%$. The precise correspondence with the actual layer widths is, however, likely to be mildly fortuitous. This is because, while self-consistently applied, the criteria used to delineate the beginning and ending of each layer is subjectively defined. Thus, it is probably true that the more relevant property associated with the empirically determined mean layer structure is the ratio of the layer widths, since this property largely removes the effect of the criteria used to identify the beginning and end of the layers. In either case, however, the properties satisfy those associated with $\phi_{c}=\Phi$ to within about $2 \%$.

\section{B. Mean Velocity Profile}

Figure 5 shows mean profile data from a wind tunnel boundary layer, pipe flow, water tunnel boundary layer, and the atmospheric surface layer. ${ }^{29}$ As indicated, employing $\kappa=$ $\Phi^{-2} \simeq 0.382$ yields convincing agreement with these data, and with previous wind tunnel 


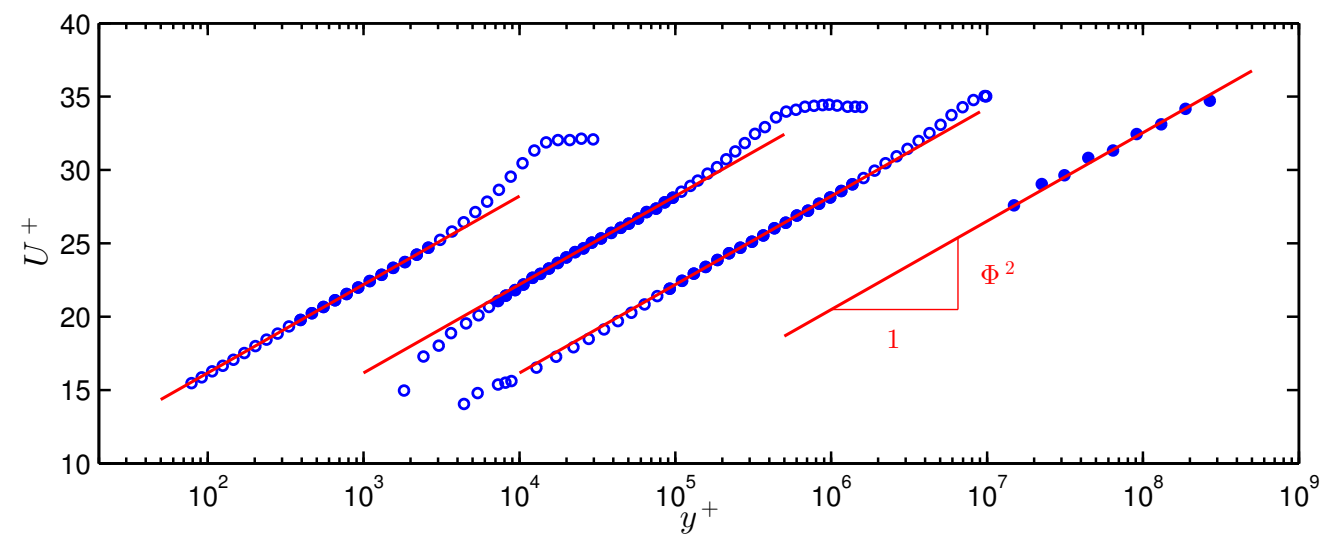

FIG. 5. Semi-logarithmic mean velocity profiles at large $\delta^{+}$reported by Marusic et al. ${ }^{29}$ Profiles from left to right are from a wind tunnel boundary layer, water channel boundary layer, pipe flow and atmospheric surface layer. The horizontal axis is shifted by one decade for each respective profile. Red lines denote $U^{+}=\Phi^{2} \ln \left(y^{+}\right)+B$. In accord with the analysis of (2), the solid symbols fall within $\Phi^{2} \sqrt{\delta^{+}} \lesssim y^{+} \lesssim C \delta^{+}$, where $C=0.15$ is employed here.

studies $^{28}$ that found $\kappa=0.384$, atmospheric surface layer measurements ${ }^{27}$ that found $\kappa=$ 0.387, recent $\delta^{+}=4080$ channel $\mathrm{DNS}^{11}$ that reports $\kappa=0.383$, and the recent analysis of high Reynolds number pipe flow ${ }^{30}$ that concluded $\kappa=0.4 \pm 0.02$. For the studies just mentioned, and the data of figure $5, \kappa=\Phi^{-2}$ agrees to within the uncertainty of the measurements.

Relative to the position of logarithmic dependence, the invariant form (5) on the layer hierarchy ensures the existence of a similarity solution for $U^{+}\left(y^{+}\right)$on the domain $\phi_{c}^{2} \sqrt{\delta^{+}} \lesssim$ $y^{+} \lesssim C \delta^{+}$, where the analytical upper bound estimate for $C$ is $\lesssim 0.5$. Empirical data and the analytically predicted similarity solution at finite $\delta^{+}$, however, indicate that the upper bound is somewhat less than this. In accord with these observations, ${ }^{29}$ the solid symbols in figure 5 are delineated using $C=0.15$. On this and subsequent figures we use $\Delta y_{I I}^{+}=\Phi \sqrt{\delta^{+}}$for the inner-normalized width of layer II (see table I), and similarly, $\Delta y_{I I}^{+}+\Delta y_{I I I}^{+}=\Phi^{2} \sqrt{\delta^{+}}=(1+\Phi) \sqrt{\delta^{+}}$. (Recall that $\Phi^{2}=\Phi+1$ and $\Phi^{-1}=\Phi-1$ are definitional properties of $\Phi$.) Note, once again, that layer III is the incipient layer on the inertial domain of interest. The similarity solution on the inertial domain is associated with a linear $W(y)$ (figures 2 and 6), and it specifies how and where a logarithmic mean profile emerges with increasing $\delta^{+}$. The data of figure 5 exhibit agreement with these analytical 
predictions. ${ }^{29,37}$

\section{Slope of $W^{+}\left(y^{+}\right)$}

While the scaling properties listed in table I and the position and slope of the logarithmic mean profile provide evidence that the value of $\alpha$ is near unity, results more specifically pertaining to the nature of the underlying self-similarities constitute a deeper level of scrutiny. One is the prediction that $d W / d y=\phi_{c}^{-1}\left(\simeq \Phi^{-1}\right)$ on the inertial domain of interest. In this regard, profile data from a $\delta^{+}=2004$ channel flow $\mathrm{DNS}^{43}$ were previously used ${ }^{36}$ to estimate $d W / d y$ (figures 2 and 6). The best estimate from this analysis indicated that $d W / d y$ $\left(=\phi_{c}^{-1}\right) \simeq 0.625 \pm 0.0027$, which corresponds to $\kappa \simeq 0.39$. The estimation procedure for $d W / d y$ was repeated for the $\delta^{+}=4080$ channel DNS $^{11}$ data shown in figure 2, yielding $d W / d y \simeq 0.62 \pm 0.002$. This corresponds to $\kappa \simeq 0.384$, which agrees closely with the above noted value of $\kappa=0.383$ previously cited for this data set. ${ }^{11}$ As discussed in $\S I I$, analysis of (2) explicitly predicts that $\kappa \rightarrow(d W / d y)^{2}=\phi_{c}^{-2}$ as $y^{+} \rightarrow \infty$. Here we note that the $\delta^{+}=2004$ DNS results agree with $\phi_{c}=\Phi$ to within about $1.1 \%$, while the $\delta^{+}=4080$ results are within about $0.4 \%$. The trend indicated in figure 6 suggests that these deviations are likely to be associated with finite $\delta^{+}$.

\section{Geometry of the Momentum Transporting Motions}

Recalling that $W^{+}$is the average size of the turbulent motions responsible for the generation of $T^{+}=-\langle u v\rangle^{+}$, we now return to the previous observation that the analyses of $(2)$ indicate that $\kappa=\phi_{c}^{-2}$ is separable into two effects. The analytical basis for this lies in the fact that the differential transformations (6) that lead to (5) alter $T^{+}$and $y^{+}$, but not $U^{+}$. More specifically, (20) holds on the inertial domain, and according to (6) there is a $\phi_{c}$ effect associated with the change in momentum with $\hat{y}$, and a counteracting $\phi_{c}$ effect on $\hat{T}$ that keeps the equation invariant. For the reasons now described, one effect is associated with (i) the size variation of the momentum transporting motions, and the other is apparently related to $(i i)$ how these motions cover the area of any given wall parallel plane.

Figure 6 shows a direct statistical measure of the changing size of the momentum transporting motions. This measure is essentially the two-dimensional spatial analogue of the 


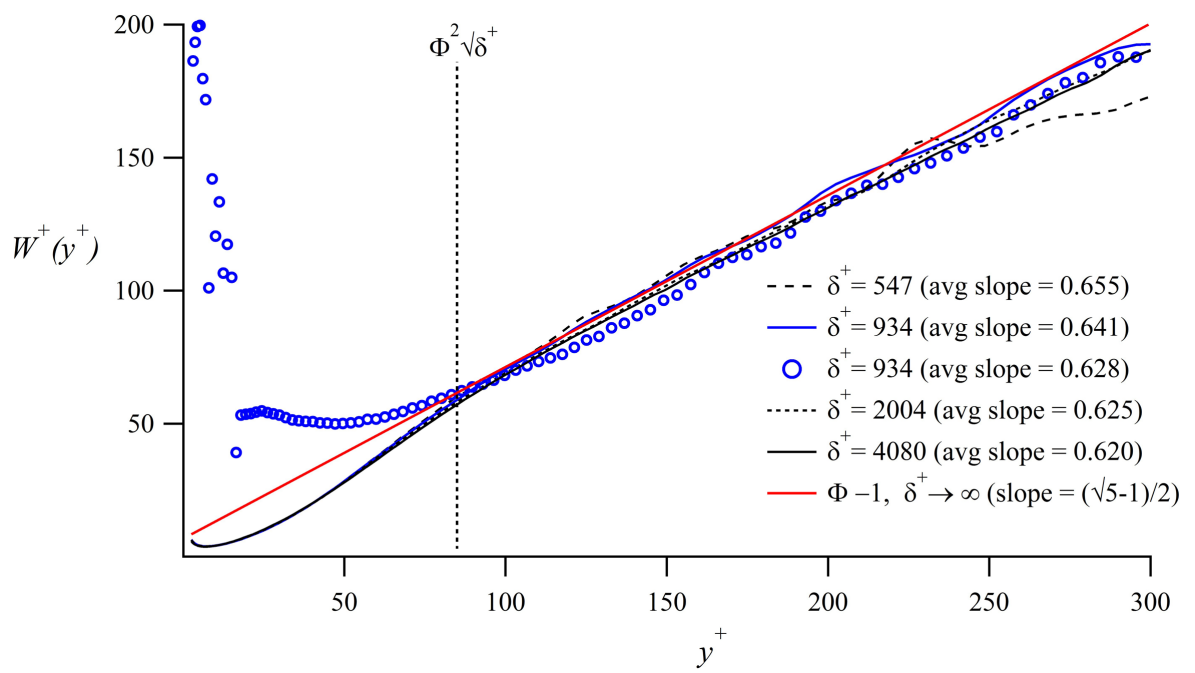

FIG. 6. Length scale distributions, $W^{+}\left(y^{+}\right)$, associated with the similarity solution to the mean momentum equation (lines), as compared with the two-dimensional zero-crossing function (circles) determined from planar slices through the instantaneous $u v<0$ motions. The vertical line denotes the onset of the inertial domain at $\delta^{+}=934$. Data are from channel DNS. ${ }^{11,43,48}$

average zero-crossing in a time series. Using data from a $\delta^{+}=934$ channel DNS, ${ }^{48}$ the regions of negative $u v$ were identified within each $(x, z)$ plane. The ensembles of these regions at each $y$ location were then aligned at their peak values and the average of this aligned ensemble was computed. The resulting shape looks something like a two dimensional probability distribution, except that the tails cross zero. Their cross-sectional shape is well-approximated by an ellipse. The desired quantity is the area, $A_{b}$, covered by the base of this object, as it provides a consistently defined measure of the size of the negative $u v$ motions. The base areas were estimated by fitting to an ellipse. The ensemble averages had a noise floor of about $\pm 2.5 \%$. Accordingly, the $A_{b}^{+}$measurements were made just above this noise floor. This apparently accounts for their slightly lower values than the corresponding $W^{+}(y)$ profile in figure 6 .

In accord with the analysis, the calculated $A_{b}^{+}\left(y^{+}\right)$profile begins to coincide with the $W^{+}\left(y^{+}\right)$profile near $y^{+}=\Phi^{2} \sqrt{\delta^{+}}$. An analysis of the vorticity field properties suggests that the onset of this behavior occurs when there is a sufficient scale separation between the relevant velocity and vorticity field motions. Under this condition (i.e., on the inertial domain), advection becomes the most significant vorticity transport mechanism. ${ }^{38}$ In the 
region interior to $y^{+}=\Phi^{2} \sqrt{\delta^{+}}$this scale separation mechanism is rationally attributable to vorticity stretching. On the inertial domain the agreement between $W^{+}\left(y^{+}\right)$and $A_{b}^{+}\left(y^{+}\right)$ is compelling, as even the detailed features of the two profiles are observed to track. This level of agreement between both the slope and magnitude of the $A_{b}^{+}$and $W^{+}$profiles is somewhat surprising, especially when one considers that $W^{+}\left(y^{+}\right)$is the inverse square root of the mean velocity profile curvature, ${ }^{35}$ while $A_{b}^{+}\left(y^{+}\right)$is derived from perhaps the simplest statistic by which to estimate the size of the motions of interest. The slope of the $A_{b}^{+}\left(y^{+}\right)$ profile is 0.628 , as quantified over the same domain used to estimate $W^{+}\left(y^{+}\right)$.

Note, however, that $d A_{b}^{+} / d y^{+}$is the derivative of an area, while $d W^{+} / d y^{+}$is the derivative of a length. This observation is reconciled by recognizing that the mean behaviors at any $y^{+}$ location result from an ensemble of interactions in the associated wall-parallel plane. Thus, we postulate a scaling argument regarding the structure in wall-parallel planes, and then invoke the analytical result that $W \rightarrow \phi_{c}^{-1} y \simeq(\Phi-1) y$ as $\delta^{+} \rightarrow \infty$.

Suppose that the momentum transporting motions are self-similarly space filling at any $y$, and as $y$ varies these motions exhibit a self-similar change in scale. This is consistent with the results of the previous section that with each change of scale in $y$ the relationship between the size of the momentum transporting motions and the space in which they exist remains unchanged. Given this, a general representation of the mean vorticity is

$$
\frac{d U}{d y}=\frac{u_{\tau}}{\ell} \frac{y \gamma}{\lambda \xi}
$$

In (28) the ratio $u_{\tau} / \ell$ represents a measure of the vorticity that takes into account spatial intermittency in any given $(x, z)$ plane, and $(y \gamma) /(\lambda \xi)$ generically allows for scale changes in the momentum transporting motions with increasing $y$. Here $\gamma, \lambda$ and $\xi$ are length scales that account for multi-directional stretching and dilatation. Equation (28) provides for an array of potentially complex changes with $y$, since $\ell, \gamma, \lambda$ and $\xi$ could each be a different function of $y$. If, however, the spatial intermittency property is self-similarly related to the scale changes in the manner indicated by the analysis, then $\ell=\gamma=\lambda=\xi=f(y)=\phi_{c}^{-1} y$, and under $(25) \phi_{c}^{-1} y=\Phi^{-1} y=(\Phi-1) y$. With this $(28)$ becomes

$$
\frac{d U}{d y}=\frac{u_{\tau}}{y(\Phi-1)^{2}}=\frac{\Phi^{2} u_{\tau}}{y}=\frac{u_{\tau}}{\kappa y}
$$

When simplified, the ratio $(y \gamma) /(\lambda \xi)$ equals $\phi_{c} \simeq \Phi$, and this provides a plausible resolu- 
tion of the issue of $d A_{b}^{+} / d y^{+}$being the derivative of an area. It implies, however, that within any $(x, z)$ plane the fraction of area coverage by the motions accounting for $T$ remains fixed at $\phi_{c}^{-1} \simeq(\Phi-1)$. This requirement reflects the invariant shape (in a statistical sense) of the relevant motions, even though these motions are continually changing size. This is the same type of geometric self-similarity exhibited by an equiangular (logarithmic) spiral. For each rotation (replicating interval) of $\theta=\pi / 2$, the radius of a logarithmic spiral undergoes a constant multiplicative increase, thus reflecting its changing size but invariant shape. In the present context, note that if $D$ is interpreted as the replicating interval, and $y_{2} / y_{1}$ as being proportional to the radius, then (16) has the same form as the equation of a logarithmic spiral. Physically, one side of (20) reflects the changing step in $\hat{y}$ required to produce a constant increment $D$ in $U^{+}$, while the other reflects the constant area coverage, in each $(x, z)$ plane, of the momentum transporting motions that produce this velocity increment. Lastly, note that this is an inherent property of the self-similar inertial region, and thus is predicted to hold even if $\phi_{c}$ is not equal to $\Phi$.

High resolution $u$ and $v$ time series data were acquired in the High Reynolds Number Boundary Layer Wind Tunnel (HRNBLWT) at the University of Melbourne and the Flow Physics Facility (FPF) at the University of New Hampshire. These facilities are described in Nickels et al. ${ }^{49}$ and Vincenti et al. ${ }^{50}$ respectively. For these measurements, the inner-normalized dimension of the $\times$-array hotwire sensor (wire length and spacing) ranged between 6 and 11 viscous units. Furthermore, owing to the small noise-floor of these measurements, no threshold was employed in the analysis.

Figure 7 shows the fraction of time that the $u v$ time series signal is negative as a function of $y^{+} / \sqrt{\delta^{+}}$for varying $\delta^{+}$. This statistic is equivalent to the area coverage of the motions responsible for the wallward transport of momentum. Plotting versus $y^{+} / \sqrt{\delta^{+}}$allows one to assess whether these data agree with the theoretical prediction that the self-similar region of interest begins near the outer edge of layer III, i.e, $y^{+}=O\left(\sqrt{\delta^{+}}\right)$, independent of $\delta^{+}$. The data indicate that this is indeed the case, and are also consistent with the $\alpha=1$ condition that this position specifically scales like $y^{+} \simeq\left(\phi_{c}+1\right) \sqrt{\delta^{+}} \simeq \Phi^{2} \sqrt{\delta^{+}}$. The Reynolds number trend in figure 7 is also in accord with the area coverage equaling $\Phi^{-1}$ as $\delta^{+} \rightarrow \infty$. A clearer quantification of this is presented in figure 8, which plots the average value of the area coverage in the region $\Phi^{2} \sqrt{\delta^{+}} \lesssim y^{+} \lesssim 0.15 \delta^{+}$versus $\delta^{+}$.

The inertial domain of interest at the highest $\delta^{+}(\simeq 10,800)$ falls between the vertical 
Self-similarity in the inertial region of wall turbulence

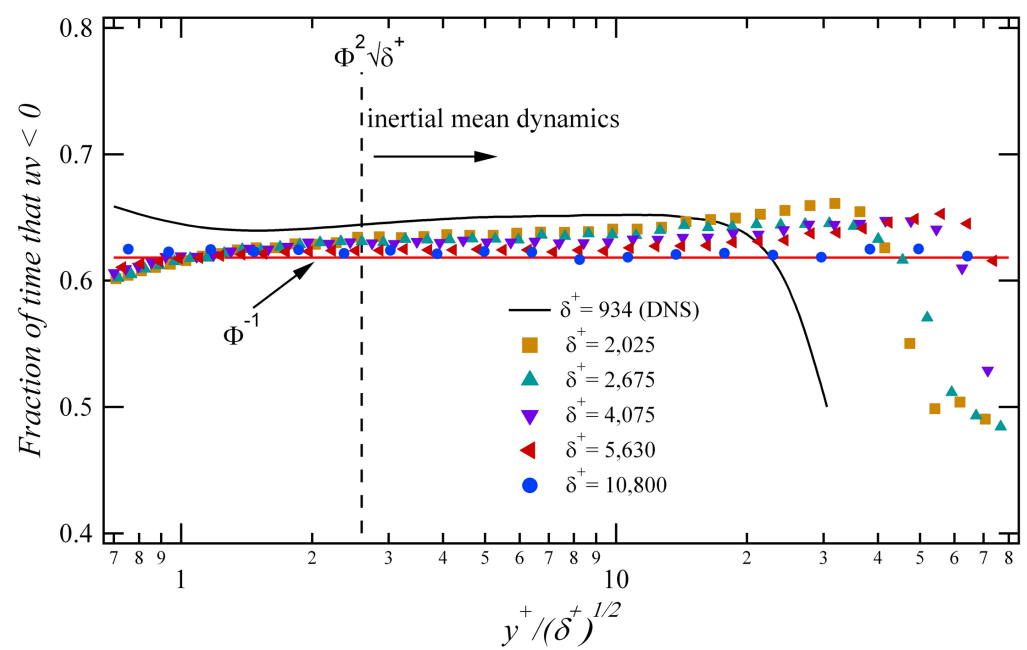

FIG. 7. Area fraction that $u v$ is negative for various $\delta^{+}$and plotted versus $y^{+} / \sqrt{\delta^{+}}$Data at $\delta^{+} \simeq 10,800$ are from the FPF, while all other measurements are from the HRNBLWT. The line is from channel DNS. ${ }^{48}$

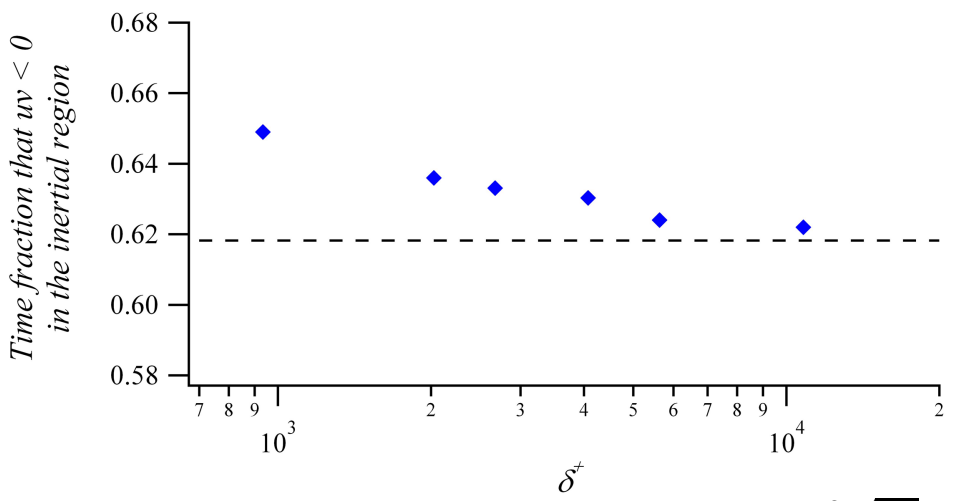

FIG. 8. The average area fraction that $u v$ is negative in the domain $\Phi^{2} \sqrt{\delta^{+}} \lesssim y^{+} \lesssim 0.15 \delta^{+}$, plotted versus $\delta^{+}$. Dashed line indicates $\Phi^{-1}$.

dashed lines in figure 9, which is also where the $U^{+}\left(y^{+}\right)$slope most convincingly approximates $\Phi^{2}$. As is apparent, the value of the area coverage plateaus in the inertial domain and approaches $\Phi^{-1}$ with increasing $\delta^{+}$. At $\delta^{+}=10,800$, the average of this value is 0.622 , which is within about $0.6 \%$ of $\Phi^{-1}=0.61803 \ldots$. The results of figures 7 and 9 also seem to suggest that the self-similar inertial domain may extend farther than $0.15 \delta$, but certainly not beyond $0.5 \delta$, which is the analytically estimated upper-bound.$^{34}$

The emerging picture is one in which $\kappa$ gains its value owing to two separate features associated with the turbulent motions responsible for momentum transport. These pertain to $(i)$ their wall normal change in scale, and $(i i)$ their invariant shape (area coverage) in any given $(x, z)$ plane. In support of the previous and present analyses, figures 7 - 9 
Self-similarity in the inertial region of wall turbulence

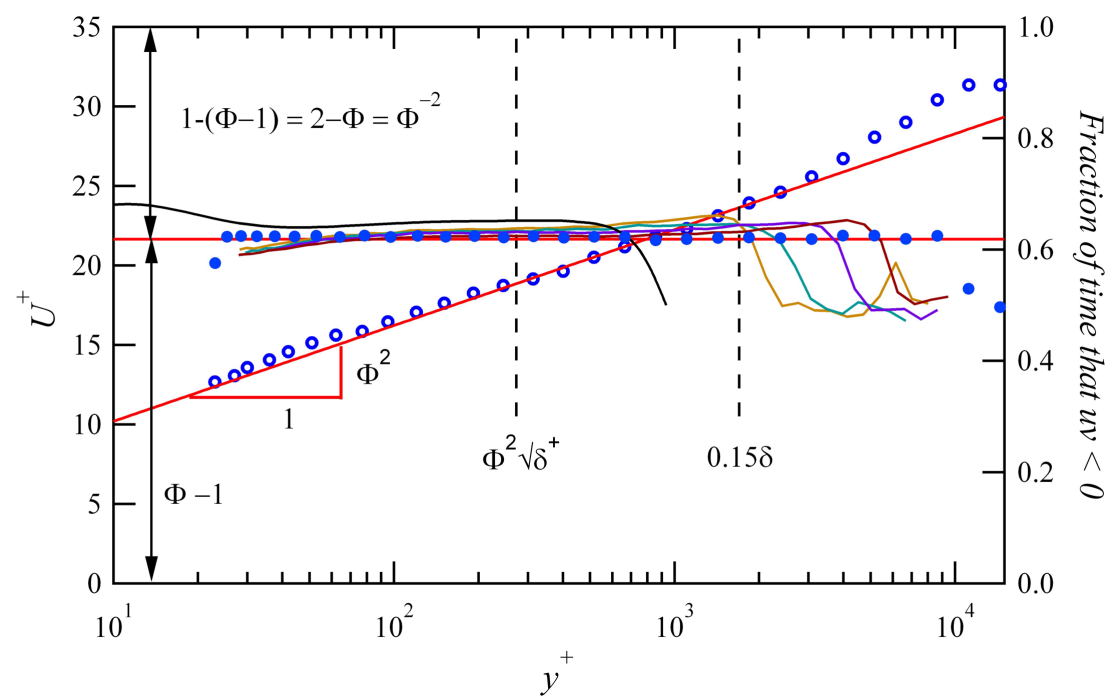

FIG. 9. Logarithmic mean velocity profile at $\delta^{+} \simeq 10,800$ (left axis, open symbols), and area fraction that $u v$ is negative (right axis, lines and solid symbols) versus $y^{+}$. Layers II and III boundaries are indicated for $\delta^{+} \simeq 10,800$. Lower $\delta^{+}$profiles (lines) are indicated in figure 7 .

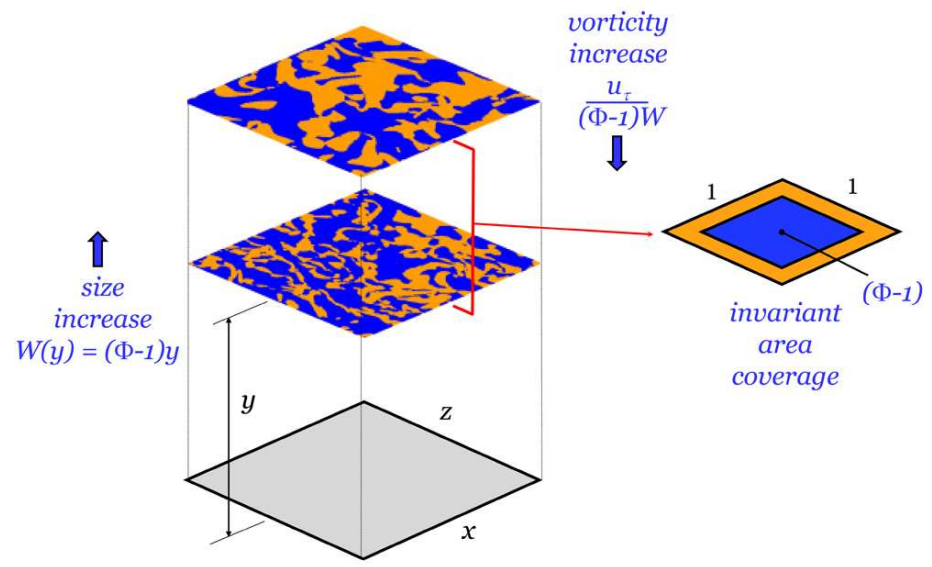

FIG. 10. Two instantaneous wall-parallel slices of the $u v$ product from channel $\mathrm{DNS}^{48}$ at $\delta^{+}=934$. Blue regions denote a negative product, while positive regions are in gold. The average of this quantity, which is negative, constitutes the net wallward flux of momentum. At finite $\delta^{+}$the area coverage of the negative (blue) regions is approximated by $\Phi^{-1}=\Phi-1$, while the average change in scale with increasing $y$ is approximated by $(\Phi-1) y$. For the low Reynolds number $\left(\delta^{+}=934\right)$ flow depicted, the Fife similarity parameter is about $\phi_{c}=0.64$, as quantified by the $d W^{+} / d y^{+}$ estimate of figure 6 . 
provide evidence that these properties directly relate to $\phi_{c}^{-1} \simeq \Phi^{-1}$. Figure 10 further clarifies the underlying geometry of the momentum transporting motions, and summarizes the underlying behaviors that pertain to the stretching transformations of $y^{+}$and $T^{+}$that yield the invariant form (5). Dynamically, the two geometric properties stem from a constant wall-normal flux of turbulent inertial force, i.e., (3). Physically, these self similar features emerge because external boundary effects on the inertial subdomain of the layer hierarchy diminish as $\delta^{+} \rightarrow \infty$. Mathematically, they arise because both $y^{+}$and $T^{+}$are stretched by the similarity transformation, while $U^{+}$is not. The structure shown in figure 10 is demonstrated herein to exhibit convincing analytical and empirical support.

\section{CONCLUSIONS AND DISCUSSION}

The analytical framework employed herein indicates that self-similar mean dynamics emerge on an interior inertial domain of turbulent wall-flows as $\delta^{+}$becomes large. These self-similar dynamics induce a self-similar geometric structure via the coordinate stretching required for the mean momentum equation to formally admit an invariant form. These behaviours are reflected in the Fife similarity parameter, $\phi$, approaching a constant, $\phi_{c}$, on the inertial domain. ${ }^{37} \mathrm{~A}$ more expansive form of geometric self-similarity was also explored. Here the self-similarity that is analytically known to exist between each layer and its position on the hierarchy also describes the relationship between adjacent layers and positions. This condition is mathematically equivalent to the von Kármán constant, $\kappa\left(=\phi_{c}^{-2}\right)$, exactly equaling $\Phi^{-2}=2-\Phi=(3-\sqrt{5}) / 2=0.381966 \ldots$. The present results also clarify why the leading order mean forces undergo a balance exchange across layer III, that, independent of $\delta^{+}$, starts at $y^{+} \simeq \Phi \sqrt{\delta^{+}}$and completes at $y^{+} \simeq(\Phi+1) \sqrt{\delta^{+}}=\Phi^{2} \sqrt{\delta^{+}}$.

The concepts describing the condition of an intermediate asymptotic limit ${ }^{45}$ seem to embody many of the characteristics associated with the mean dynamical structure of the inertial region. Generically, an intermediate asymptotic state is attained on an interior portion of the solution domain, where the direct boundary condition (or for temporal problems, initial condition) influences diminish as the relevant parameters become infinitely large or small. For the present flow, the inertial domain necessarily resides sufficiently 'far' from the viscous and integral length scales, $\nu / u_{\tau}$ and $\delta$, respectively. In such problems, however, the boundary condition scales leave a signature that colours the solution properties, and 
consistently, the intermediate asymptotic solution reflects how the governing dynamics most naturally accommodate these remotely felt constraints. For the present problem, a geometric description of how the signature of the wall boundary condition may colour the scaling behavior of the self-similar inertial domain is given in Appendix D.

Physically, the layer hierarchy exists to realize the outward transport of mean vorticity and the inward transport mean momentum that occur simultaneously across the scales of motion between the viscous and integral scales. ${ }^{51}$ Thus, relative to the influence of the wall boundary condition, in flows over aerodynamically rough surfaces it is rational to expect that the signature of the roughness scales will always be retained on the inertial domain - albeit, in many cases subtly, e.g., see Mehdi et al. ${ }^{52}$ The conditions under which such influences become detectable on the inertial domain speak to the validity of Townsend' ${ }^{22}$ wall similarity hypothesis.

Lastly, it seems appropriate to briefly comment on how the challenges associated with precisely ascertaining the asymptotic value of $\kappa$ might eventually be resolved. The results from the study of Bailey et al. ${ }^{30}$ are relevant to this issue, as they rather convincingly indicate that an experimentally based determination of $\kappa$ (e.g., by fitting to (1)) to an accuracy characteristic of other empirically determined physical constants is unlikely to be realized in the foreseeable future. Thus, a convincing value for $\kappa$ seems more likely to arise by first gaining a deeper understanding of what this quantity physically represents and how the physics associated with it fit within a well-founded theoretical description of wall-flow dynamics. In this regard, the present theoretical framework is believed to make a contribution. For example, among its findings the present theory indicates that $\kappa$ is dynamically associated with the asymptotic constancy of the flux of turbulent inertial force across an intermediate range of scales. ${ }^{51}$ As evidenced by the data of figures 6 and 7 this description reveals that $\kappa$ has relevance to analytically determined geometric properties relating to both the mean velocity and the Reynolds stress - a notion that gains credence given that both $U^{+}$and $T^{+}$are solutions to (2). As additional properties relating to $\kappa$ are revealed, greater clarity regarding the value of von Kármán's constant, and the self-similar dynamics underlying its behaviors, will be realized. 


\section{ACKNOWLEDGMENT}

This work was supported by the Australian Research Council and the National Science Foundation funded New Hampshire EPSCoR program. The authors are grateful to the researchers who made their DNS data available via the web.

\section{APPENDIX A: LOGARITHMIC DEPENDENCE OF $U^{+}$}

Equation 9 holds exactly if $A$ is a constant, and approximately if $A$ is approximately constant. ${ }^{33}$ Here we arrive at (9) by treating $A$ as a constant, which holds as $\delta^{+} \rightarrow \infty$. To cast (2) in a form that exposes its dependence on the layer hierarchy, we employ the transformation

$$
T_{\beta}^{+}=T^{+}\left(y^{+}\right)+\frac{y^{+}}{\delta^{+}}-\beta y^{+} .
$$

Differentiation of (30) with respect to $y^{+}$yields,

$$
\frac{d T_{\beta}^{+}}{d y^{+}}=\frac{d T^{+}}{d y^{+}}+\frac{1}{\delta^{+}}-\beta .
$$

Inserting (31) into (2) yields

$$
0=\beta+\frac{d^{2} U^{+}}{d y^{+2}}+\frac{d T_{\beta}^{+}}{d y^{+}},
$$

which is still an exact representation of the mean force balance. Transformation of (32) using (6) yields the invariant form (5).

Note that

$$
\frac{d T_{\beta}^{+}}{d y^{+}}\left(y_{\beta}^{+}\right)=\frac{d T^{+}}{d y^{+}}\left(y_{\beta}^{+}\right)-\beta=0
$$

locates the position of each layer on the hierarchy as depicted in figure 3 . Differentiating (33) with respect to $\beta$,

$$
\frac{d^{2} T^{+}}{d y^{+2}}\left(y_{\beta}^{+}\right) \frac{d y_{\beta}^{+}}{d \beta}-1=0,
$$

and employing the definition of $A$ in (3) yields, 
Self-similarity in the inertial region of wall turbulence

$$
\frac{d y_{\beta}^{+}}{d \beta}=-A^{-1} \beta^{-3 / 2}
$$

Because $A$ is treated as a constant, (35) may be integrated to obtain

$$
y_{\beta}^{+}=-A^{-1} \int \beta^{-3 / 2} d \beta=2 A^{-1} \beta^{-1 / 2}+C,
$$

where $C$ is the same constant that appears in (9) and (10). Recognizing that $W^{+}=\beta^{-1 / 2}$ and that each $y_{\beta}^{+}$uniquely corresponds to $y^{+}$on the hierarchy domain, one can see that (36) recovers (10). Using (36) to solve for $\beta$ in terms of $y^{+}$and $A$, and then equating this to the definition of $\beta$ given in (3) yields

$$
\frac{d T^{+}}{d y^{+}}=(2 / A)^{2}\left[\left(y^{+}-C\right)^{-2}-\left(y_{m}^{+}-C\right)^{-2}\right]=\frac{\phi^{2}}{\left(y^{+}-C\right)^{2}}-\frac{\phi^{2}}{\left(y_{m}^{+}-C\right)^{2}} .
$$

In (37) the last term on the right derives from $d T^{+} / d y^{+}=0$ at the position, $y_{m}^{+}$, where $T^{+}$ attains its maximum value, $T_{m}^{+}$. Integration of (37) gives

$$
T^{+}\left(y^{+}\right)=C^{\prime}-\frac{\phi^{2}}{y^{+}-C}-\frac{y^{+} \phi^{2}}{\left(y_{m}^{+}-C\right)^{2}},
$$

where $C^{\prime}$ approaches a constant as $\delta^{+} \rightarrow \infty$ (Ref. 33, p. 955), also see Appendix B below.

Equation 38 can then be inserted into the once-integrated form of (2)

$$
\frac{d U^{+}}{d y^{+}}=1-T^{+}-\frac{y^{+}}{\delta^{+}}
$$

By noting that $\phi \rightarrow \phi_{c}$ as $\delta^{+} \rightarrow \infty$ and requiring that the derivatives of $U^{+}$vanish as $y^{+} \rightarrow$ $\infty$, integration of (39) yields (9). Finally note that one could also choose the asymptotic relation for $T^{+}$first (see Appendix B below). Its substitution into (39) directly recovers (19), and the subsequent integration yields (1).

\section{APPENDIX B: ASYMPTOTIC POSITION OF MAXIMUM $T^{+}\left(y^{+}\right)$}

The theoretical framework described in $\S \mathrm{II}$ yields the equation for $T^{+}\left(y^{+}\right)$given by (38). Empirical evidence, ${ }^{53,54}$ asymptotic approximations ${ }^{55}$ and direct analysis ${ }^{32}$ of (2) establish that the position of maximum $T^{+}$is given by $y_{m}^{+}=\lambda_{m} \sqrt{\delta^{+}}$, where $\lambda_{m} \rightarrow$ const. as $\delta^{+} \rightarrow \infty$. These same observations and analyses also indicate that $T_{m}^{+} \rightarrow 1$ as $\delta^{+} \rightarrow \infty$.

Neglecting $C$ for large $\delta^{+}$, and using $y_{m}^{+}=\lambda_{m} \sqrt{\delta^{+}}$gives 


$$
T^{+}\left(y^{+}\right)=C^{\prime}-\frac{\phi^{2}}{y^{+}}-\frac{y^{+} \phi^{2}}{\lambda_{m}^{2} \delta^{+}} .
$$

Noting that $T^{+}\left(\delta^{+}\right)=0$, evaluation of (40) as $\delta^{+} \rightarrow \infty$ yields,

$$
C^{\prime}=\frac{\phi_{c}^{2}}{\lambda_{m}^{2}}
$$

Similarly, evaluation of $(40)$ at $y_{m}^{+}$gives

$$
1=\frac{\phi^{2}}{\lambda_{m}^{2}}-\frac{\phi^{2}}{\lambda_{m} \sqrt{\delta^{+}}}-\frac{\phi^{2} \sqrt{\delta^{+}}}{\lambda_{m} \delta^{+}},
$$

and thus $\lambda_{m} \rightarrow \phi_{c}$ like $1 / \sqrt{\delta^{+}}$as $\delta^{+} \rightarrow \infty$, e.g., Ref. 34 .

Here we note some observations. One is that with $\kappa=\phi_{c}^{-2}$ (e.g., as reflected in the asymptotic form of equation 9), one obtains $\lambda_{m}=\kappa^{-1 / 2}$. This recovers the result that Afzal $^{55}$ found using his mesolayer theory. To the authors' knowledge, Afzal was the first

to recognize the importance of the intermediate length, $\sqrt{\nu \delta / u_{\tau}}$, relative to the scaling properties of (39). A second observation is that with $\phi_{c}=\Phi$ (or for that matter $\phi_{c} \simeq 0.39$ ) one sees that the asymptotic value of $\lambda_{m}$ is $\simeq 1.6$. Comparison reveals that this value is close to the thickness of layer II, see table I. This further corroborates the asymptotic geometric representation of the layer hierarchy depicted in figure 4. Lastly, we note that the approach of $\lambda_{m}$ to $\phi_{c}$ is relatively slow, $\sim 1 / \sqrt{\delta^{+}} \rightarrow 0$. Physically, $\lambda_{m}=\phi_{c}$ is attained when the finite sheet of mean vorticity between the wall and $y_{m}^{+}$compresses to an infinitesimal thickness relative to $\delta^{+}$, see Ref. 39. Note that this is also the rate at which the $O\left(\nu / u_{\tau}\right)$ thickness of layer I compresses to infinitesimal thickness relative to the thickness of layer II.

\section{APPENDIX C: PROBLEMS EQUIVALENT TO DEMONSTRATING THAT $\kappa=\Phi^{-2}$}

As indicated in $\S$ III C, analytically determining that $\alpha=1$ is equivalent to demonstrating that $\kappa=\Phi^{-2}$. This task is nontrivial in part because the domain of interest gains its selfsimilar behaviors as boundary condition effects become remote. This apparently thwarts the direct use of boundary conditions to determine $\phi_{c}$. Here we briefly pose three equivalent problems that, if solved, analytically demonstrate that $\kappa=\Phi^{-2}$. 
The $W_{i}$ and associated $y_{i}$ are each described by geometric sequences. Asymptotically, the common ratio in each of these sequences is known to be $\left(\phi_{c}+1\right) / \phi_{c}$, while $y_{i} / W_{i}=\phi_{c}$. Analytically demonstrating that $y_{i+1} / y_{i} \rightarrow y_{i} / W_{i}$ for large $i$ would equivalently demonstrate that $\phi_{c}=\Phi$.

It is well known that the ratio of the successive terms, $F_{n+1} / F_{n}$, of the Fibonacci sequence converges to $\Phi$. It is less well known that the ratio of successive terms of any sequence that satisfies $S_{n+2}=S_{n}+S_{n+1}$ also converges to $\Phi$, e.g., see Livio. ${ }^{56}$ Demonstrating that either the $W_{i}$ or the $y_{i}$ satisfy this relation guarantees that $\phi_{c} \rightarrow \Phi$ as the number of hierarchy layers becomes large, i.e., as $\delta^{+} \rightarrow \infty$.

From (7) note that $\hat{y}=\beta^{1 / 2}\left(y^{+}-y_{\beta}^{+}\right)$. Inserting this into (20) yields

$$
\frac{d U^{+}}{d \hat{y}}=\frac{\phi_{c}^{2}}{\hat{y}}
$$

With attention restricted to the $\phi \rightarrow \phi_{c}$ portion of the hierarchy, the integration of (43) yields $D$. Furthermore, if the limits of this integral can be chosen such that

$$
D=\phi_{c}^{2} \ln \phi_{c}
$$

then inserting (44) into (16) yields $\phi_{c}=\Phi$. Thus, demonstrating the validity of (44) achieves the desired result. Relative to this, examination of the integral on the incipient inertial layer (layer III) helps clarify the conditions that restrict the integration to the $\phi \rightarrow \phi_{c}$ portion of the layer hierarchy. In the limit, the start of layer III is located at $y^{+}=\phi_{c} \sqrt{\delta^{+}}$(Appendix B). Thus, while the lower limit of the domain of interest tends to infinity as $\delta^{+} \rightarrow \infty$, the position $y^{+}=\phi_{c} \sqrt{\delta^{+}}$simultaneously tends to zero relative to $\delta^{+}$.

\section{APPENDIX D: ASYMPTOTIC GEOMETRY OF THE WALL-FLOW HIERARCHY}

The results of $\S$ III reveal that the layer-to-layer transitions on the hierarchy are described by a geometric sequence having a common ratio equal to $\phi_{c} \simeq \Phi=(\Phi+1) / \Phi$. One property of a geometric sequence is that any given member is, by definition, the geometric mean of the surrounding adjacent members, and by extension, the geometric mean of members that are $n=1,2,3, \ldots \infty$ times removed. This is exemplified in figure 11 for a geometric sequence with common ratio equal to 2 , where 
Self-similarity in the inertial region of wall turbulence

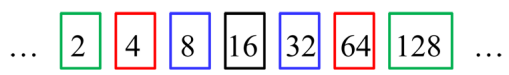

FIG. 11. Segment of a geometric sequence having common ratio equal to 2. Note that the center point (in this case arbitrary) is, by definition, the geometric mean of the sequence members located at $\pm n$ steps away from that point.

$$
16=\sqrt{8 \times 32}=\sqrt{4 \times 64}=\sqrt{2 \times 128}=\ldots
$$

Layer III (table I) is the central layer of the hierarchy as its inner-normalized width is the geometric mean of adjacent $W^{+}$layers (as just described), and its anchor position, $y_{m}^{+}$, within the flow is the geometric mean position of the $y_{\beta}^{+}$, Ref. 47. For a continuous layer hierarchy there is no apparent way to a priori pinpoint the central layer from intermediate layers on the hierarchy. One can, however, use the end points, $y_{p i}$ and $y_{p o}$ (see, figure 2) to estimate $y_{m}^{+}$, i.e.,

$$
y_{m}^{+} \simeq \sqrt{y_{p i}^{+} y_{p o}^{+}} .
$$

As documented for both channel and boundary layer flows, ${ }^{47,57}$ the physical origin of this construction begins during the transitional regime. Here a hierarchy of momentum sources and sinks (motions respectively bearing positive and negative $d T / d y$ interior to and beyond the initial peak in $T$ ) begins to form. The net source motions spread inward toward the wall, and the net sink motions spread outward toward the channel/pipe center or free stream. Eventually, this two-way spread of TI toward the periphery becomes constrained by the boundary conditions. This marks the onset of the four layer regime, and simultaneously, when $\nu / u_{\tau}$ and $\delta$ become parameters relevant to scaling turbulent wall-flows. This also marks when a nascent inertial region beyond the outer edge of layer III first appears.

As described by Barenblatt, ${ }^{45}$ in problems where there exists an intermediate asymptotic state, the factors relating to the boundary conditions (and the scales they impose) continue to leave their imprint on the solution. Thus, the present results lead to the expectation that the end points of the hierarchy can also be expressed in terms of $\kappa \simeq \Phi^{-2}$ as $\delta^{+} \rightarrow \infty$. With this in mind, we note that the finite $\delta^{+}$estimate for $y_{p i}^{+} \simeq 7$ is within about $0.15 u_{\tau} / \nu$ of $y^{+}=\kappa^{-2} \simeq 6.85$, and that $y^{+}=\kappa \delta^{+} \simeq 0.382 \delta^{+}$is within about $0.12 \delta^{+}$of the estimate for $y_{p o}^{+}$. Note further that through the use of (46) these estimates yield, 


$$
y_{m}^{+}=\sqrt{\kappa^{-2} \kappa \delta^{+}}=\sqrt{\kappa^{-1} \delta^{+}}=\phi_{c} \sqrt{\delta^{+}},
$$

which is the asymptotic value for $y_{m}^{+}$found in Appendix B.

The second equality in (47) indicates that the construction just described allows one further step in scale factor $\kappa$ toward the periphery, while still preserving the asymptotic result (47). Namely, $\phi_{c} \sqrt{\delta^{+}}$is the geometric mean of the inner-normalized integral scale, $\delta^{+}$, and the thickness of layer I, $y^{+}=\kappa^{-1} \simeq 2.6$. As described in the Appendix of Wei et al., ${ }^{58}$ the methodology used to reveal the asymptotic scaling behaviors between $y_{p i}$ and $y_{p o}$ can also be applied as the wall is approached. In this case, however, the characteristic length is $\nu / u_{\tau}$, and the resulting invariant equation is

$$
\frac{d^{2} \hat{U}}{d y^{+2}}+\frac{d \hat{T}}{d y^{+}}+1=0
$$

Unlike in (5), the individual terms in (48) are only known right at the wall. Here the pressure gradient is identically equal to the mean viscous force (flux of mean spanwise vorticity). This prevents developing other constraints, such as the velocity increment across the layer, which was used to advantage in the analysis of (5). From (48), however, one can surmise that the initial nonlinear variations in $U^{+}$and $T^{+}$account for the behavior of the mean layer (layer I) that is immediately adjacent to the wall. ${ }^{58}$ This leads one to suspect that these variations are the ultimate origin of the boundary length scale signature on the inertial layer.

\section{REFERENCES}

${ }^{1}$ Tennekes, H. \& Lumley, J.L. 1972 A First Course in Turbulence. MIT Press, Cambridge, MA.

${ }^{2}$ Pope, S.B. 2000 Turbulent Flows. Cambridge University Press, Cambridge.

${ }^{3}$ Davidson, P.A. 2000 Turbulence: An Introduction for Scientists and Engineers. Oxford University Press, Oxford.

${ }^{4}$ Marusic, I., McKeon, B.J., Monkewitz, P.A., Nagib, H.M., Smits, A.J. \& Sreenivasan, K.R. 2010 Wall-bounded turbulent flows: Recent advances and key issues. Phys. Fluids 22, 065103. 
${ }^{5}$ KLEWICKI, J. 2010 Reynolds number dependence, scaling, and dynamics of turbulent boundary layers. J. Fluids Eng., 132, 094001.

${ }^{6}$ Smits, A.J., McKeon, B.J. \& Marusic, I. 2011 High Reynolds Number Wall Turbulence. Ann. Rev. Fluid Mech. 43, 353-375.

${ }^{7}$ PrandtL, L. 1925 Bericht uber die Entstehung der Turbulenz. Z. Angew. Math. Mech. 5, 136-139.

${ }^{8}$ VOn KÁrmán, T. 1930 Mechanische Ahnlichkeit und Turbulenz. Nachr. Ges. Wiss. Goettingen, Math.-Phys. Kl. 1, 58-76.

${ }^{9}$ Bradshaw, P. 1974 Possible origin of Prandt's mixing-length theory. Nature 249, 135136.

${ }^{10}$ Mizuno, Y. \& Jimenez, J. 2011 Mean velocity and length-scales in the overlap region of wall-bounded turbulent flows. Phys. Fluids. 23, 085112.

${ }^{11}$ Pirozzoli, S. 2014 Revisiting the mixing-length hypothesis in the outer part of turbulent wall layers: mean flow and wall friction. J. Fluid Mech. 745, 378-397.

${ }^{12}$ Millikan, C. 1939 A critical discussion of turbulent flows in channels and circular tubes. Proceedings of the Fifth International Congress of Applied Mechanics. J. D. Hartog and H. Peters, eds., Wiley, New York, 5772-5776.

${ }^{13}$ Yajnik, K. 1970 Asymptotic Theory of Turbulent Shear Flows. J. Fluid Mech. 42, 411427.

${ }^{14}$ Mellor, G.L. 1972 The large Reynolds number asymptotic theory of turbulent boundary layers. Int. J. Eng. Sci. 10, 851-873.

${ }^{15}$ Barenblatt, G.I., Chorin, A.J. \& Prostokishin, A. 1997 Scaling laws for fully developed turbulent flow in pipes. Appl. Mech. Rev., 50, 413-429.

${ }^{16}$ Walker, J.D.A. 1998 Turbulent boundary layers II: Further developments. Recent Advances in Boundary Layer Theory (CISM courses and lectures). A. Kluwick, ed., SpringerVerlag, Vienna, 390, 145-230.

${ }^{17}$ Wosnik, M., Castillo, L. \& George, W.K. 2000 A theory for turbulent pipe and channel flows. J. Fluid Mech. 421, 115-145.

${ }^{18}$ AfZAL, N. 2001 Power law and log law velocity profiles in fully developed turbulent boundary layer flow: equivalent relations at large Reynolds number. Acta Mech., 151, $171-183$.

${ }^{19}$ Panton, R. 2005 Review of Wall Turbulence Described by Composite Expansions. Appl. 
Self-similarity in the inertial region of wall turbulence

Mech. Rev., 58, 1-36.

${ }^{20}$ Monkewitz, P.A., Chauhan, K.A. \& Nagib, H.M. 2008 Comparison of mean flow similarity laws in zero pressure gradient turbulent boundary layers. Phys. Fluids 20, 105102.

${ }^{21}$ Landau, L.D. \& Lifshitz, E.M. 1944 Mekhanika Sploshnykh Sred. Gostekhizdat, Moscow.

${ }^{22}$ Townsend, A. 1976 The Structure of Turbulent Shear Flow. Cambridge University Press, Cambridge.

${ }^{23}$ Perry, A. \& Chong, M. 1982 On the mechanism of wall turbulence. J. Fluid Mech. 119, 173-217.

${ }^{24}$ Perry, A., Henbest, S. \& Chong, M. 1986 A theoretical and experimental study of wall turbulence. J. Fluid Mech. 218, 405-438.

${ }^{25}$ Perry, A. \& Marusic, I. 1995 A wall-wake model for the turbulence structure of boundary layers. Part 1. Extension of the attached eddy hypothesis. J. Fluid Mech. 298, $361-388$

${ }^{26}$ Zagarola, M.V. \& Smits, A.J. 1998 Mean-flow scaling of turbulent pipe flow. J. Fluid Mech. 373, 33-79.

${ }^{27}$ Andreas, E.L., Claffey, K.J., Jordan, R.E., Fairall, C.W., Guest, P.S., Persson, P.O.G., \& Grachev, A.A. 2006 Evaluations of the von Kármán constant in the atmospheric surface layer. J. Fluid Mech., 559, 117-149.

${ }^{28}$ Nagib, H. \& Chauhan, K. 2008 Variation of von Kármán coefficient in canonical flows. Phys. Fluids 20, 101518.

${ }^{29}$ Marusic, I., Monty, J., Hultmark, M. \& Smits, A. 2013 On the logarithmic region in wall turbulence. J. Fluid Mech., 716, R3-11.

${ }^{30}$ Bailey, S.C.C., Vallikivi, M., Hultmark, M. \& Smits, A.J. 2014 Estimating the value of von Kármán's constant in tubulent pipe flow. J. Fluid Mech., 749, 79-98.

${ }^{31}$ Zeff, B.W., Kleber, B., Fineberg, J. \& Lathrop, D.P. 2000 Singularity dynamics in curvature collapse and jet eruption on a fluid surface. Nature, 403, 401-404.

${ }^{32}$ Wei, T., Fife, P., Klewicki, J. \& McMurtry, P. 2005 Properties of the mean momentum balance in turbulent boundary layer, pipe and channel flows. J. Fluid Mech. 522, 303-327.

${ }^{33}$ Fife, P., Klewicki, J., McMurtry, P. \& Wei, T. 2005a Multiscaling in the presence 
Self-similarity in the inertial region of wall turbulence

of indeterminacy: Wall-induced turbulence. Multiscale Modeling and Simulation 4, 936959.

${ }^{34}$ Fife, P., Wei, T., Klewicki, J. \& McMurtry, P. 2005b Stress gradient balance layers and scale hierarchies in wall-bounded turbulent flows. J. Fluid Mech. 532, 165-189.

${ }^{35}$ Fife, P., Klewicki, J. \& Wei, T. 2009 Time averaging in turbulence settings may reveal an infinite hierarchy of length scales. J. of Discrete and Continuous Dynamical Systems, 24, 781-807.

${ }^{36}$ Klewicki, J., Fife, P. \& Wei, T. 2009 On the logarithmic mean profile. J. Fluid Mech., 638, 73-93.

${ }^{37}$ KLEwiCKI, J. 2013a Self-similar mean dynamics in turbulent wall flows. J. Fluid Mech., 718, 596-621.

${ }^{38}$ KLEWICKI, J. 2013b A description of turbulent wall-flow vorticity consistent with mean dynamics. J. Fluid Mech., 737, 176-204.

${ }^{39}$ KLEWICKI, J. 2013c On the singular nature of turbulent boundary layers. Procedia IUTAM, 9, 69-78.

${ }^{40}$ Blasius, H. 1908 Grenzschichten in Flssigkeiten mit kleiner Reibung. Z. Math. Phys., $56,1-37$.

${ }^{41} \mathrm{Wu}, \mathrm{X} .$, \& Moin, P. 2008 A direct numerical simulation study on the mean velocity characteristics in turbulent pipe flow. J. Fluid Mech. 608, 81-112.

${ }^{42}$ Abe, H, Kawamura, H. \& Matsuo, Y. 2004 Surface heat-flux fluctuations in a turbulent channel flow up to $R e_{\tau}=1020$ with $\operatorname{Pr}=0.025$ and 0.71 . Int. J. Heat and Fluid Flow 25, 404-419.

${ }^{43}$ Hoyas, S. \& Jimenez, J. 2006 Scaling the velocity fluctuations in turbulent channels up to $R e_{\tau}=2003$. Phys. Fluids 18, 011702.

${ }^{44}$ Hansen, A. 1964 Similarity Analysis of Boundary Value Problems in Engineering. Prentice-Hall, New York.

${ }^{45}$ Barenblatt, G.I. 1996 Scaling, Self-similarity, and Intermediate Asymptotics. Cambridge University Press, Cambridge.

${ }^{46}$ Meneveau, C. \& Marusic, I. 2013 Generalized logarithmic law for high-order moments in turbulent boundary layers. J. Fluid Mech., 719, R1-11.

${ }^{47}$ Klewicki, J., EBner, R. \& Wu, X. 2011 Mean dynamics of transitional boundary-layer flow. J. Fluid Mech., 682, 617-651. 
${ }^{48}$ Del Alamo, J.C., Jimenez, J., Zandonade, P. \& Moser, R. 2004 Scaling of the energy spectra of turbulent channels. J. Fluid Mech., 500, 135-144.

${ }^{49}$ Nickels, T.B., Marusic, I., Hafez, S., Hutchins, N. \& Chong, M.S. 2007 Some predictions of the attached eddy model for a high Reynolds number boundary layer. Phil. Trans. Roy. Soc. A, 365, 907-822.

${ }^{50}$ Vincenti, P., Klewicki, J., Morrill-Winter, C., White, C. \& Wosnik, M. 2013 Streamwise velocity statistics in turbulent boundary layers that spatially develop to high Reynolds number. Exp. Fluids 54, 1629-1638.

${ }^{51}$ Klewicki, J., Fife, P., Wei, T. \& McMurtry, P. 2007 A physical model of the turbulent boundary layer consonant with mean momentum balance structure. Phil. Trans. Roy. Soc. A 365, 823-839.

${ }^{52}$ Mehdi, F., Klewicki, J. \& White, C. 2013 Mean force structure and its scaling in rough-wall turbulent boundary layers. J. Fluid Mech. 731, 682-712.

${ }^{53}$ Long, R.R. \& Chen, T.-C. 1981 Experimental evidence for the existence of the mesolayer in turbulent systems. J. Fluid Mech. 105, 19-59.

${ }^{54}$ Sreenivasan, K.R. \& Sahay, A. 1997 The persistence of viscous effects in the overlap region, and the mean velocity in turbulent pipe and channel flows. In Self-Sustaining Mechanisms of Wall Turbulence (ed. R. Panton), pp. 253-272. Computational Mechanics Publications, Southampton, UK.

${ }^{55}$ AfzAL, N. 1982 Fully developed turbulent flow in a pipe: An intermediate layer. Ing.Arch. 52, 355-377.

${ }^{56}$ Livio, M. 2002 The Golden Ratio: The Story of Phi, the World's Most Astonishing Number . Broadway Books, New York.

${ }^{57}$ Elsnab, J., Klewicki, J., Maynes, D \& Ameel, T. 2011 Mean dynamics of transitional channel flow. J. Fluid Mech. 678, 451-481.

${ }^{58}$ Wei, T., Fife, P. \& Klewicki, J. 2007 On scaling the mean momentum balance and its solutions in turbulent Couette-Poiseuille flow. J. Fluid Mech. 573, 371-398. 